\title{
Correlated thermoelectrics
}

\author{
V. Zlatić \\ Institute of Physics, Bijenicka c. 46, P. O. B. 304,1000 Zagreb,Croatia
}

\begin{abstract}
In the first part of these lecture notes we introduce the phenomenological equations for describing the heat and charge transport in thermoelectric samples. We discuss the solution obtained for various boundary conditions that are appropriate for the homogeneous and inhomogeneous thermoelectrics. In the second part we develop the formalism for a linear-response many-body description of the transport properties of correlated electrons. By properly determining the local heat-current operator we show that the Jonson-Mahan theorem applies to the Hamiltonians that are commonly used for the intermetallic compounds with Cerium, Europium and Ytterbium ions, so the various thermal-transport coefficient integrands are related by powers of frequency. We illustrate how to use this formalism by calculating the thermoelectric properties of the periodic Anderson model and, then, show that these results explain the experimental data on heavy fermions and valence fluctuators. Finally, we calculate the thermoelectric properties of the Falicov-Kimball model and use the results to explain the anomalous features of the intermetallic compounds in which one observes the valence-change transition.
\end{abstract}

Keywords: thermoelectricity, electron correlations

PACS: $71.27 .+\mathrm{a}, 72.15 \mathrm{Jf}, 72.20 \mathrm{~Pa}$

\section{INTRODUCTION}

We are interested in the processes that occur in a thermoelectric sample connected to two reservoirs on different temperatures, chemical potentials, and voltage. The temperature difference, $\Delta T$, and the difference in the voltage and chemical potentials, $\Delta V$ and $\Delta \mu$, give rise to the charge and energy transport through the sample that would bring, eventually, both reservoirs into equilibrium. For a given thermoelectric material and the boundary condition defined by $\Delta T, \Delta \mu$, and $\Delta V$ we ask what is the distribution of temperature, electrical and chemical potential in the sample and how are the current densities related to the gradients $\nabla T(\mathbf{x}), \nabla \phi(\mathbf{x})$ and $\nabla \mu(\mathbf{x})$, where $\phi(\mathbf{x})$ and $\mu(\mathbf{x})$ are the electrical and chemical potential, respectively. These questions can be answered by solving the transport equations, together with the continuity equations and the Maxwell equation. The transport equations relate the currents to the driving forces, which are assumed to be given by a linear combinations of the gradients $\nabla T(\mathbf{x}), \nabla \phi(\mathbf{x})$ and $\nabla \mu(\mathbf{x})$. The transport coefficients are specified by the thermoelectric properties of the sample and can either be obtained from the measurements or calculated from a microscopic model. The choice of the currents and the forces is not unique, i.e., the transport equations can be written in a number of equivalent ways. Here, we consider only the pairs that lead to the symmetric transport coefficients. In what follows, we derive the compleat set of equations for the thermoelectric problem and discuss the solution. We neglect any changes in the thermodynamic state of the reservoirs and assume that each elementary (macroscopic) volume of the sample can be described by local thermodynamic functions. In our case, the lo- 
cal quantities describe a system of interacting particles in the presence of an external potential.

The currents generated by the driving forces give rise to irreversible processes that increase the entropy of the thermoelectric sample. The rate of change of the entropy is obtained from the integral[1,2]

$$
S=\int d^{3} \mathbf{x} s(u, n)
$$

where $s(\mathbf{x})$ is the local entropy density, $n(\mathbf{x})$ particle density, $u(\mathbf{x})$ the energy density, and the integration is over the sample. Using the first law of thermodynamics,

$$
d s=\frac{\partial s}{\partial u} d u+\frac{\partial s}{\partial n} d n
$$

where $\partial s / \partial u=1 / T$ and $\partial s / \partial n=-\mu / T$, we can write the rate of change of entropy as

$$
\frac{d S}{d t}=\int d^{3} x\left\{\frac{1}{T} \frac{d u}{d t}-\frac{\mu}{T} \frac{d n}{d t}\right\}
$$

The conservation of charge and energy provide the continuity equations that relate the time-derivatives of the charge and energy densities to the divergencies of the corresponding current vectors,

$$
\frac{d n}{d t}=-\frac{1}{e} \nabla \cdot \mathbf{J}
$$

and

$$
\frac{d u}{d t}=-\nabla \cdot \mathbf{J}_{u}
$$

where $\mathbf{J}(\mathbf{x})$ and $\mathbf{J}_{u}(\mathbf{x})$ are the charge current density and the energy current density, respectively. The continuity equation can also be written in terms of the internal energy current density, $\mathbf{J}_{E}=\mathbf{J}_{u}-\phi \mathbf{J}$,

$$
\frac{d u}{d t}+\nabla \cdot \mathbf{J}_{E}=\mathbf{E} \cdot \mathbf{J}
$$

where $\mathbf{E}=-\nabla \phi$ is the external field. In a stationary state, where $d u / d t=0$, the total energy current is conserved, $\nabla \cdot \mathbf{J}_{u}=0$, while $\nabla \cdot \mathbf{J}_{E}$ is equal to the work $\mathbf{E} \cdot \mathbf{J}$ which is done in the system by the external field per unit volume and time.

Using the continuity equations and substituting $\nabla \cdot \mathbf{J}_{E}=T \nabla\left(\mathbf{J}_{E} / T\right)-T \mathbf{J}_{E} \nabla(1 / T)$ and $(\mu / T) \nabla \cdot \mathbf{J}=\nabla(\mu \mathbf{J} / T)-\mathbf{J} \nabla(\mu / T)$ we rewrite Eq.(3) in the form

$$
\frac{d S}{d t}=\int d^{3} x \frac{1}{T}\left\{\mathbf{J} \cdot\left(\mathbf{E}-\frac{T}{e} \nabla \frac{\mu}{T}\right)+\mathbf{J}_{E} \cdot T \nabla \frac{1}{T}\right\}-\int d^{3} x \nabla \cdot \frac{\mathbf{J}_{E}-\mu \mathbf{J} / e}{T}
$$

that allows a simple interpretation[1, 2]. The second term can be trqansformed into a surface integral which gives the entropy change arising from an entropy current flowing across the boundary of the sample. Inroducing the heat current density $\mathbf{J}_{Q}=\mathbf{J}_{E}-\mu \mathbf{J} / e$ 
and the entropy current density $\mathbf{J}_{S}=\mathbf{J}_{Q} / T$, the rate of change of the entropy due to these boundary currents can be written as,

$$
-\int d^{3} x \nabla \cdot \frac{\mathbf{J}_{Q}}{T}=-\int_{\text {surface }} d \mathbf{A} \cdot \mathbf{J}_{S} .
$$

The first term in Eq. (7) gives the rate of change of the local entropy density at each point of the sample, as described by the expression,

$$
T \frac{d s_{V}}{d t}=\mathbf{J} \cdot\left(\mathbf{E}-\frac{T}{e} \nabla \frac{\mu}{T}\right)+\mathbf{J}_{E} \cdot T \nabla \frac{1}{T} .
$$

In a stationary state, the forces and currents are time-independent and all the entropy produced locally is transported out of the sample by the entropy current.

The charge and energy conservation allow us to express the rate of change of the local entropy density as a scalar product

$$
T \frac{d s_{V}}{d t}=\mathbf{J} \cdot \mathbf{x}_{\phi}^{L}+\mathbf{J}_{E} \cdot \mathbf{x}_{E}^{L},
$$

where $\mathbf{J}$ and $\mathbf{J}_{E}$ are the currents corresponding to the generalized forces,

$$
\begin{aligned}
\mathbf{x}_{\phi}^{L} & =-\nabla \phi-\frac{T}{e} \nabla \frac{\mu}{T}=-\nabla\left(\phi+\frac{\mu}{e}\right)-\frac{\mu T}{e} \nabla \frac{1}{T}, \\
\mathbf{x}_{T}^{L} & =T \nabla \frac{1}{T} .
\end{aligned}
$$

Following Onsager, we introduce the generalized current vector

$$
\hat{\mathbf{J}}=\left(J^{x}, J^{y}, J^{z}, J_{E}^{x}, J_{E}^{y}, J_{E}^{z}\right)=\left(\hat{J}_{1}, \ldots, \hat{J}_{6}\right),
$$

and the generalized force vector

$$
\hat{\mathbf{x}}=\left(x_{x}^{\phi}, x_{y}^{\phi}, x_{z}^{\phi}, x_{x}^{T}, x_{y}^{T}, x_{z}^{T}\right)=\left(\hat{x}_{1}, \ldots, \hat{x}_{6}\right),
$$

such that

$$
\frac{d s_{V}}{d t}=\frac{\hat{\mathbf{J}} \cdot \hat{\mathbf{x}}}{T} .
$$

Finally, assuming that the system is close to an overall equilibrium, and that the generalized forces are small, we approximate the current vectors by a linearized form,

$$
\hat{J_{a}}=\sum_{b} L_{a b} \hat{x}_{b}
$$

The general arguments of irreversible statistical physics show that one can define the currents and the forces in a number of ways but as long as the scalar product in Eq. (14) is conserved the transport coefficients are symmetric and satisfy the Onsager relations $[1,2,3]$.

$$
L_{a b}=L_{b a} .
$$


Note, the transport equations and the continuity equation are not sufficient for a unique determination of the current vectors and the scalar fields $T(\mathbf{x}), \phi(\mathbf{x})$ and $\mu(\mathbf{x})$ for a given set of boundary conditions. To obtain the complete solution we have to relate the electrical potential to the charge and current densities by the Maxwell equations. For a non-magnetic sample without the displacement currents these are

$$
\begin{array}{ll}
\nabla \cdot \mathbf{E}=4 \pi \rho_{T} & \nabla \times \mathbf{E}=-\frac{1}{c} \frac{\partial \mathbf{B}}{\partial t}, \\
\nabla \cdot \mathbf{B}=0 & \nabla \times \mathbf{B}=\frac{4 \pi}{c} \mathbf{J}^{T},
\end{array}
$$

where $\rho^{T}=\rho+\rho_{\text {ext }}$ and $\mathbf{J}^{T}=\mathbf{J}+\mathbf{J}_{\text {ext }}$ are the total charge and current densities, $\rho_{\text {ext }}$ and $\mathbf{J}_{\text {ext }}$ are the external charged and current densities, and $\rho=e\left(n-n_{0}\right)$, where $n_{0}$ is the equilibrium number density of electrons in the sample.

We now have a complete set of equations and can find the solution of the thermoelectric problem by the following procedure. For an arbitrary choice of $T(\mathbf{x}), \phi(\mathbf{x})$ and $\mu(\mathbf{x})$ find the local values of $n(\mathbf{x})$ from the partition function and obtain the current densities $\mathbf{J}$ and $\mathbf{J}_{u}$ from the transport equations. Use Maxwell equations to obtain a relationship between $\mathbf{E}(\mathbf{x}), \mathbf{B}(\mathbf{x}), n(\mathbf{x})$ and $\mathbf{J}(\mathbf{x})$, and solve these equations to determine the electrical field for a given value of $T(\mathbf{x})$ and $\mu(\mathbf{x})$. Alternatively, if no current is flowing, determine the electrical potential for a given charge distribution which depends on $T(\mathbf{x})$ and $\mu(\mathbf{x})$ from the Poisson equation. Finally, use the continuity equations to find the distribution of temperatures and the chemical potential in the sample. The particular solution obtained in such a way depends on the choice of transport coefficients (they describe the sample) and on the boundary conditions (they describes the reservoirs).

\section{PHENOMENOLOGICAL EQUATIONS}

The irreversible thermodynamics[1,3] deals with the systems subject to a thermal gradient, and the inhomogeneities in the electrical and chemical potential. The inhomogeneities of $\mu(\mathbf{x})$ are due to it deviation from the uniform chemical potential $\mu_{0}$ that we use to control the total number of particles in the sample. The electro-chemical potential which drives the currents in the absence of the temperature gradient is $\bar{\phi}=\phi+\mu / e$; the electro-chemical field is $\bar{E}=-\nabla(\phi+\mu / e)$.

The pair $\left(\mathbf{J}, \mathbf{J}_{E}\right)$ and $\left(\mathbf{x}_{\phi}^{L}, \mathbf{x}_{T}^{L}\right)$ which we introduced before is convenient because the coefficients $L_{a b}$ can be calculated for a given Hamiltonian by the linear response theory. The phenomenological transport equations read,

$$
\begin{aligned}
\mathbf{J}(x) & =L_{11} \mathbf{x}_{\phi}^{L}+L_{12} \mathbf{x}_{T}^{L}, \\
\mathbf{J}_{E}(x) & =L_{21} \mathbf{x}_{\phi}^{L}+L_{22} \mathbf{x}_{T}^{L},
\end{aligned}
$$

and the corresponding continuity equation is

$$
\nabla \cdot \mathbf{J}_{E}=\mathbf{E} \cdot \mathbf{J}
$$


which shows that the Joule heat is the "source" of the energy current. The transport coefficients in these equations have been used by Lutinger [2] to relate the phenomenological description of heat and charge transport to microscopic calculations.

Mahan[4] redefines the coefficients and the forces as $\bar{L}_{i j}=T L_{i j}$ and $\mathbf{x}^{L}=\mathbf{x}^{M} / T$, such that

$$
\begin{aligned}
\mathbf{x}_{c}^{M} & =-\frac{\nabla \phi}{T}-\frac{1}{e} \nabla \frac{\mu}{T} \\
\mathbf{x}_{T}^{M} & =\nabla \frac{1}{T} .
\end{aligned}
$$

Since we have $\bar{L}_{i j} \mathbf{x}^{M}=L_{i j} \mathbf{x}^{L}$, the scalar product $\overline{\mathbf{J}} \cdot \overline{\mathbf{x}}$ is obviously conserved and the transport equations read

$$
\begin{aligned}
\mathbf{J}(x) & =-\bar{L}_{11}\left[\frac{\nabla \phi}{T}+\frac{1}{e} \nabla \frac{\mu}{T}\right]+\bar{L}_{12} \nabla \frac{1}{T}, \\
\mathbf{J}_{E}(x) & =-\bar{L}_{21}\left[\frac{\nabla \phi}{T}+\frac{1}{e} \nabla \frac{\mu}{T}\right]+\bar{L}_{22} \nabla \frac{1}{T} .
\end{aligned}
$$

The coefficients $\bar{L}_{i j}$ are also convenient for relating the phenomenological theory to microscopic calculations[4].

In addition to the charge current density $\mathbf{J}$ and the energy current density $\mathbf{J}_{E}$ one often considers other pairs of currents, like the number current density $\mathbf{J}_{N}=\mathbf{J} / e$ and the heat current density $\mathbf{J}_{Q}=\mathbf{J}_{E}-(\mu / e) \mathbf{J}=\mathbf{J}_{u}-\bar{\phi} \mathbf{J}$ or the entropy current density $\mathbf{J}_{S}=\mathbf{J}_{Q} / T$ or the total energy current density $\mathbf{J}_{u}$. In a non-interacting system, $\mathbf{J}_{Q}=\mathbf{J}_{u}-\bar{\phi} \mathbf{J}$ is fieldfree, i.e., $\mathbf{J}_{Q}=\mathbf{J}_{K}$ describes the kinetic energy current. 'This agrees with the intuitive notion of the heat (current) as a kinetic energy current' [1].

Most applications use transport equations with the charge current $\mathbf{J}$ and the heat current $\mathbf{J}_{Q}=T \mathbf{J}_{S}$. The condition

$$
e \mathbf{J}_{N}(x) \mathbf{x}_{c}^{G}+T \mathbf{J}_{S}(x) \mathbf{x}_{Q}^{G}=-\mathbf{J}_{N}(x) \nabla \bar{\mu}(x)-\mathbf{J}_{S}(x) \nabla T(x),
$$

gives the generalized forces

$$
\begin{aligned}
\mathbf{x}_{c}^{G} & =-\frac{\nabla \bar{\mu}}{e}=-\nabla \bar{\phi} \\
\mathbf{x}_{Q}^{G} & =-\frac{1}{T} \nabla T,
\end{aligned}
$$

where $\bar{\mu}=e \bar{\phi}$. The transport equations are

$$
\begin{gathered}
\mathbf{J}(x)=-G_{11} \nabla \bar{\phi}(x)-G_{12} \frac{\nabla T(x)}{T}, \\
\mathbf{J}_{Q}(x)=-G_{21} \nabla \bar{\phi}(x)-G_{22} \frac{\nabla T(x)}{T}
\end{gathered}
$$


and the coefficients $G_{i j}$ satisfy Onsager relation. Equating the currents in Eqs.(23) and (19) we find $G_{11}=L_{11}$ and $G_{12}=L_{12}-(\mu / e) L_{11}$. Equating $\mathbf{J}_{E}(x)=\mathbf{J}_{Q}(x)$ for $\mathbf{J}=0$, and using $\left.\nabla \bar{\phi}=\left(G_{12} / G_{11}\right) \nabla T / T\right)$, gives $G_{22}=L_{22}-2(\mu / e) L_{12}-(\mu / e)^{2} L_{11}$.

The continuity equation for the heat current density $q(x, t)$ is

$$
\frac{d q}{d t}+\nabla \cdot \mathbf{J}_{Q}=\overline{\mathbf{E}} \cdot \mathbf{J}
$$

Callen[1] redefines the coefficients in (23) as $N_{i j}=T G_{i j}$ and the forces as

$$
\begin{aligned}
\mathbf{x}_{c}^{N} & =-\frac{\nabla \bar{\phi}}{T} \\
\mathbf{x}_{Q}^{N} & =-\frac{1}{T^{2}} \nabla T=\nabla \frac{1}{T} .
\end{aligned}
$$

The transport equations now read

$$
\begin{aligned}
\mathbf{J}(x) & =-\frac{N_{11}}{T} \nabla \bar{\phi}-\frac{N_{12}}{T^{2}} \nabla T \\
\mathbf{J}_{Q}(x) & =-\frac{N_{21}}{T} \nabla \bar{\phi}-\frac{N_{22}}{T^{2}} \nabla T .
\end{aligned}
$$

Another pair[4] that is often used for a system of particles moving in an external field $-\nabla \phi(\mathbf{x})$ is the charge current $\mathbf{J}(x)$ and the total energy current $\mathbf{J}_{u}(x)=\mathbf{J}_{Q}(x)+\bar{\phi} \mathbf{J}(x)$. The invariance of the scalar product

$$
\mathbf{J} \mathbf{x}_{c}^{P}+\mathbf{J}_{u} \mathbf{x}_{u}^{P}=-\mathbf{J} \frac{\nabla \bar{\phi}}{T}+\mathbf{J}_{Q} \nabla \frac{1}{T}
$$

gives the generalized forces

$$
\begin{aligned}
& \mathbf{x}_{c}^{P}=-\nabla \frac{\bar{\phi}}{T} \\
& \mathbf{x}_{u}^{P}=\nabla \frac{1}{T} .
\end{aligned}
$$

The transport equations are

$$
\begin{aligned}
\mathbf{J}(x) & =-P_{11} \nabla \frac{\bar{\phi}}{T}+P_{12} \nabla \frac{1}{T} \\
\mathbf{J}_{u}(x) & =-P_{21} \nabla \frac{\bar{\phi}}{T}-P_{22} \nabla \frac{1}{T} .
\end{aligned}
$$

The coefficients $P_{i j}$ satisfy Onsager's relations and are related to $L_{i j}$ or $\bar{L}_{i j}$ in an obvious way. 


\section{PHYSICAL INTERPRETATION}

Transport equations describe the properties of a sample connected to the reservoirs at different temperatures and electro-chemical potentials, such that the charge and heat currents flow from one reservoir to the other. The currents flow in response to the external perturbation, so as to bring the system in the equilibrium. Since the driving fields are given by the gradients of potentials, the particles move towards the potential minimum.

Physical interpretation of the transport equations is particularly simple if we define a new set of transport coefficients in the following way: $\sigma=G_{11}, \sigma \alpha=G_{12} / T, \beta=G_{21}$, and $\bar{\kappa}=G_{22} / T$. The transport equations then $\operatorname{read}[5]$,

$$
\begin{aligned}
\mathbf{J}(x) & =-\sigma \nabla \bar{\phi}-\sigma \alpha \nabla T, \\
\mathbf{J}_{Q}(x) & =-\beta \nabla \bar{\phi}-\bar{\kappa} \nabla T,
\end{aligned}
$$

where $\sigma$ can be interpreted as the electrical conductivity and $\alpha$ as the Seebeck coefficient. However, $\nabla \bar{\phi}$ and $\nabla T$ are not the correct generalized forces for the currents $\mathbf{J}(x)$ and $\mathbf{J}_{Q}(x)$ and the symmetry between transport coefficients is lost, i.e., $\beta \neq \sigma \alpha$ $(\beta=\sigma \alpha T)$. The first part of the heat current in Eq.(28) arises because the electrons experience a change in the velocity when there are local inhomogeneities in the electrical potential. In a stationary flow, this additional kinetic energy is converted into reversible thermoelectric heat. The second term of the heat current is due to the diffusion of particles from the high to low temperatures. The overall entropy production can be written as[5],

$$
\frac{d S}{d t}=\int\left[\frac{\kappa(\nabla T)^{2}}{T^{2}}+\frac{\mathbf{J}^{2}}{\sigma T}\right] d V \geq 0,
$$

where the thermoelectric term has been eliminated by partial integration. The thermoelectric effects are reversible and do not change the total entropy, even though they produce the local heat and transfer the entropy from one part of the system to another. The overall entropy production is manifestly positive-definite.

Transport Eqs.(28) together with Maxwell equation (18) and the continuity equations (4) and (5) determine completely the electrodynamics of a conductor in which the currents flow due to the electrical and thermal fields. The solution provides the distribution of electrical fields, temperature, current and heat current in the sample for any value of kinetic coefficients $\sigma, \alpha, \Pi$ and $\kappa$, and for any value of the external potential drop $\Delta V$ and temperature difference $\Delta T$. These coefficients can either be calculated from the Hamiltonian or taken as material-specific constants. In microscopic calculations we expresse the coefficients in Eqs.(28) through the Luttinger coefficients which are directly related to the correlation functions of a given microscopic model. We have $\sigma=L_{11}$ for the conductivity,

$$
\kappa=\frac{1}{T}\left(L_{22}-\frac{L_{12}^{2}}{L_{11}}\right)
$$

for the thermal conductivity, and

$$
\alpha=\frac{1}{e T}\left(\frac{L_{12} / e}{L_{11} / e^{2}}-\mu\right)
$$


for the Seebeck coefficient.

The analysis of thermoelectric effects simplifies if we write the charge and heat currents as[5],

$$
\begin{aligned}
\mathbf{J}(x) & =\sigma(\overline{\mathbf{E}}-\alpha \nabla T) \\
\mathbf{J}_{Q}(x) & =\Pi \mathbf{J}(x)-\kappa \nabla T,
\end{aligned}
$$

where $\Pi=\alpha T$ is the Peltier coefficient and $\kappa=\alpha \beta-\bar{\kappa}$ is the thermal conductivity.

Thermoelectric heat. The total energy generated per unit volume and unit time is $\dot{u}=-\nabla \cdot \mathbf{J}_{u}$. Using $\mathbf{J}_{Q}=\mathbf{J}_{u}-\bar{\phi} \mathbf{J}$, together with Eqs.(31) and (30), gives

$$
\dot{u}=\frac{\mathbf{J}^{2}}{\sigma}+\operatorname{div}(\kappa \nabla T)-T \mathbf{J} \nabla \alpha .
$$

The first term gives the rate at wich the heat is produced by the Joule heating, the second term gives the change due to the heat flowing down the temperature gradient, and the third part gives the change due to the thermoelectric heating $\dot{u}_{T E}=\mathbf{j} T \nabla \alpha$. In a stationary flow, $\dot{u}=0$, the above equation determines the temperature profile in the sample.

Ohm's law. In the absence of the temperature gradient, the current flowing in a homogeneous sample, such that $\nabla \alpha=0$, obeys the Ohm's law $\mathbf{J}=-\sigma \nabla \phi$. The current flowwing in an external field gives rise to the energy dissipation which is given by the Joule heat $\dot{q}=\mathbf{J}^{2} / \sigma$. Unless taken away by an energy current, this heating would increase the local temperature.

Thermal conductivity in an open circuit. I the absence of the charge current, Eq.(31) gives

$$
\mathbf{J}_{Q}(x)=-\kappa \nabla T(x),
$$

which is the Fourier's law for the heat flow due to temperature gradient. In a homogeneous sample, this flow generates the energy dissipation $\operatorname{div}(\kappa \nabla T)$. For a linear temperature profile in a uniform sample, such that $\nabla^{2} T=0$, and $\dot{q}=\operatorname{div}(\kappa \nabla T)=0$. linear temperature profile leads to the heat conduction which does not change the entropy of the electron gas.

Thomson heat. In a uniform conductor the coefficient $\alpha$ depends on $x$ only through temperature, so that $\nabla \alpha=(d \alpha / d T) \nabla T$. Thus, the current flowing through a uniform conductor in the presence of a temperature gradient $\nabla T$ generates the Thomson heat,

$$
\dot{q}_{T}=-\mathbf{J} \rho \nabla T .
$$

where the Thomson coefficient $\rho=-T d \alpha / d T$. Contrary to the irreversible Joule heat, the Thomson heat is linear in $\mathbf{J}$ and can be either positive or negative. 
Peltier heat. The Peltier effect is produced when the current crosses an interface between two different materials, say 1 and 2. In the absence of a temperature gradient Eq.(32) shows that the heat dissipated on the interface is

$$
\dot{q}_{\Pi}=-T \mathbf{J} \cdot \nabla \alpha,
$$

where we neglected $\mathbf{J}^{2}$ with respect to $\mathbf{J}$. Integrating over an infinitesimally thin volume element that contains the interface, and using Gauss theorem, gives the heat density generated by the current passing through the interface,

$$
\mathbf{J}_{\Pi}=\mathbf{J}_{12}
$$

where

$$
\Pi_{12}=-T\left(\alpha_{2}-\alpha_{1}\right)
$$

is the Peltier coefficient, and $\alpha(2)$ and $\alpha(1)$ are the Seebeck coefficients of the material to the left and to the right of the interface. Since the sign of $\mathbf{J}$ is arbitrary, the current flowing through a thermoelectric element at a constant temperature will dissipate or absorb the heat as it crosses the interface. Eq. (36) follows from the Onsager's relation. The Peltier and Thomson coefficients are related,

$$
\frac{\rho_{2}-\rho_{1}}{T}=-\frac{d\left(\Pi_{12} / T\right)}{d T} .
$$

The Peltier heat is due to the fact that the entropy of $n$ electrons which cross the interface is not the same on the two sides. Even for non-interacting electrons, the structure of the energy levels is different in two metals, so that the partition functions and the entropies of $n$ electrons crossing from one metal to the other, in unit time, are different on two sides. Thus, the electrons or holes absorb or emit some heat as they cross the interface and get thermally distributed over available energy levels.

What happens if the heat current flowing through the interface and $\nabla T \neq 0$ ? Because the temperature, the electrochemical potential, the normal components of the heat current and electrical current, are all continuous at the interface, Eq.(32) gives in a stationary state,

$$
J_{x} T\left(\alpha_{2}-\alpha_{1}\right)-\left.\kappa \frac{d T}{d x}\right|_{1} ^{2}=0 .
$$

If we neglect the Thompson and Joule heat, the heat current entering the interface is $\left.\kappa \frac{d T}{d x}\right|_{1}$ and the heat current leaving the interface is $\left.\kappa \frac{d T}{d x}\right|_{2}$. The heat current densities at the two sides of the interface differ by $\kappa\left(d T /\left.d x\right|_{2}-d T /\left.d x\right|_{1}\right)=j_{x} \Pi_{12}$, i.e., they differ by the Peltier heat given away or taken at the interface in unit time.

Thermoelectric power. In the stationary state, the temperature distribution in the sample is obtained from the condition $\mathbf{J}^{2} / \sigma+\operatorname{div}(\kappa \nabla T)-T \mathbf{J} \nabla \alpha=0$, the electrical field $\mathbf{E}(x)$ follows from the transport equation, and the total potential drop across the sample is given by the integral

$$
\Delta V=\int_{0}^{a} d x \mathbf{E}(x)=\int_{0}^{a} d x\left[\frac{\mathbf{J}}{\sigma}+\alpha \nabla T(x)\right] .
$$


In an open thermoelectric circuit, as the one used for measuring the Seebeck coefficient, we have $\mathbf{J}=0$ and obtain the electromotive force between the end-points $a$ and $b$ as,

$$
\mathscr{E}_{T}=\int_{a}^{b} d x \alpha(T) \frac{d T}{d x}=\int_{T(a)}^{T(c)} d T \alpha_{2}(T)-\int_{T(b)}^{T(c)} d T \alpha_{1}(T)
$$

$\alpha_{1}(T)$ and $\alpha_{2}(T)$ are the Seebeck coefficients of metals 1 and 2, and $T(a), T(b)$, and $T(c)$ are temperatures at the points $a, b$, and $c$, respectively. If $T(a)=T(b)=T$ and $\Delta T=T(c)-T(a)$ is small, then

$$
\mathscr{E}_{T}=\int_{T}^{T+\Delta T} d T\left(\alpha_{2}-\alpha_{1}\right) \simeq \Delta T\left(\alpha_{2}(T)-\alpha_{1}(T)\right) .
$$

The Seebeck coefficient is defined by the ratio $\Delta V / \Delta T$, where $\Delta V=\mathscr{E}_{T}$. For constant $\alpha(x)$, we have simply $\Delta V=\alpha \Delta T$. In a thermoelectric power generator one has to consider the case $\mathbf{J} \neq 0$.

The Seebeck voltage appears because the charged particles diffuse from the hot to the cold end and the imbalance of charge gives rise to a potential gradient across the sample. In a quasiparticle picture, the electrical energy required to transfer $n$ electrons from the hot end to the cold end against the voltage $\Delta V$ is balanced by the change in thermal energy (that is, the heat). Neglecting the shift of the quantum states due to the external potential, we approximate $n e \Delta V \simeq S \Delta T$, where $n$ is the particle density and $S$ the entropy density of the charge carriers. This gives the approximate relationship between the Seebeck coefficient and the entropy as

$$
\alpha(T)=\frac{S(T)}{e n} .
$$

\section{SOLUTION OF PHENOMENOLGICAL TRANSPORT EQUATIONS}

The heat and charge transport in a given thermoelectric device are described by the phenomenological transport equations

$$
\begin{gathered}
\mathbf{J}=\sigma \mathbf{E}-\sigma \alpha \nabla T, \\
\mathbf{J}_{Q}=\alpha T \mathbf{J}-\kappa \nabla T,
\end{gathered}
$$

that have to be solved together with the continuity equations for the charge and energy densities,

$$
\begin{gathered}
\frac{d n}{d t}=-\nabla \cdot \mathbf{J} \\
\frac{d u}{d t}=-\nabla \cdot \mathbf{J}_{u},
\end{gathered}
$$

and Maxwell equations for the electrical and magnetic fields. The particular solution of these equations describes a thermoelectric device that can operate as a refrigerator or a power generator, depending on the boundary (or the initial) condition. 
Simple thermoelectric device. Consider, as an example, a stationary state of a system shown in the figure. We want the distribution of temperatures, electrical field, and the currents in the sample of length $a$ and unit cross-section $A$, which is connected to the heat and charge reservoirs at points $x=0$ and $x=a$. The first reservoir is at temperature $T_{0}$, and the second one at $T_{A}$. The sample is characterized by transport coefficients $\sigma, \alpha$, and $\kappa$. The boundary between the sample and the reservoirs is considered to be 'ideal', i.e., without any effect on thermal transport. Magnetic effects are neglected.

For a given current $\mathbf{J}$, temperature $T_{A}$ and $T_{0}$, we find $T(x), \mathbf{E}(x)$, and the heat current $\mathbf{J}_{Q}(x)$ in the sample. If we specify the cooling rate at the source (the heat leaving the source in unit time defines $\mathbf{J}_{Q}$ in Eq.(44), we can determine $T_{0}$ for each $\mathbf{J}$ and $T_{A}$.

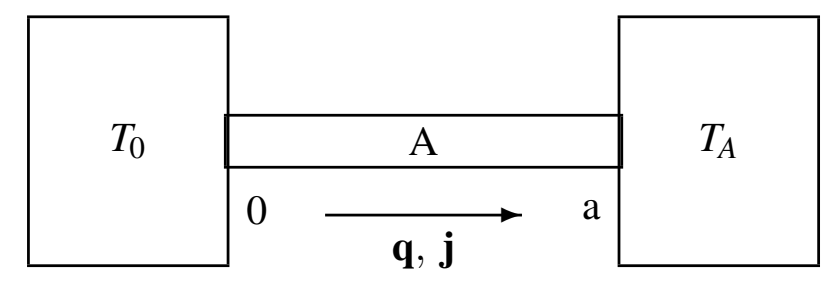

Temperature distribution. In a stationary state, the energy produced in unit time in volume $d^{3} \mathbf{x}$ around point $\mathbf{x}$ is transported out of that volume by the energy current. The temperature profile of the stationary state is defined by the continuity equation $-\nabla \mathbf{J}_{u}=-\nabla \mathbf{J}_{Q}+\mathbf{J} \cdot \mathbf{E}=0$. This leads to the Domenicali equation,

$$
\operatorname{div}(\kappa \nabla T)+\frac{\mathbf{J}^{2}}{\sigma}-T \mathbf{J} \nabla \alpha=0
$$

which has to be solved together with the boundary condition $T(0)=T_{0}$ and $T(a)=T_{A}$.

In a uniform system $\alpha(x)$ depends on $x$ only through temperature, such that $\nabla \alpha=$ $(d \alpha / d T) \nabla T$, which simplifies Eq.(47) to

$$
-\operatorname{div}(\kappa \nabla T)=\frac{\mathbf{J}^{2}}{\sigma}-\mathbf{J} \frac{d \alpha}{d T} T \nabla T
$$

The last term is the Thompson heat generated in unit time and $d \alpha / d T$ is the Thompson coefficient. The integration of Eq.(48) gives, for constant $\sigma$,

$$
-\kappa \nabla T(x)=\frac{\mathbf{J}^{2}}{\sigma} x+F-\frac{\mathbf{J}}{2} \frac{d \alpha}{d T} T^{2}(x)
$$

and, for constant $\kappa$,

$$
-\kappa T(x)=\frac{\mathbf{J}^{2}}{\sigma} \frac{x^{2}}{2}+F x+G-\frac{\mathbf{J}}{2} \frac{d \alpha}{d T} \int_{0}^{x} T^{2}(x),
$$

where the integration constants in Eq.(48) are to be determined from the boundary condition for $T(x)$ at $x=0$ and $x=l_{a}$. In what follows, we consider two limiting cases, 
which can be solved analytically. First, we keep the Joule heat and neglect the Thompson heat. Second, we neglect the Joule heat and keep the Thompson heat. In the general case, the $T(x)$ can be obtained by iteration, and the lowest order iterative solution is quite simple.

If we neglect the Thompson heat, the boundary values $T(0)=T_{0}$ and $T(a)=T_{A}$ give

$$
G=-\kappa T_{0}
$$

and

$$
F=-K\left(T_{A}-T_{0}\right)-R \frac{\mathbf{J}^{2}}{2},
$$

where $K=\kappa / l_{a}$ and $R=l_{a} / \sigma$ are the thermal conductance and electrical resistance, respectively. (The sample is of a unit crossection.) From Eqs.(44) and (49), we can find the cooling rate at the source for given $\mathbf{J}$ and $\Delta T=T_{0}-T_{a}$.

Alternatively, rather that using the boundary value for $T(x)$, we can use the initial condition for the thermal current, $\left.\mathbf{J}_{Q}(x)\right|_{x=0}=\mathbf{q}_{0}$, and find $F$ from Eqs.(44) and (49) at $x=0$,

$$
F=-\left.\kappa \nabla T\right|_{x=0}=\left(\mathbf{q}_{0}-\alpha T_{0} \mathbf{J}\right) .
$$

Together with $\left.T(x)\right|_{x=a}=T_{a}$ and Eq.(50) this provides temperature $T_{0}$ at the source.

The slope of $T(x)$, for $0 \leq x \leq l_{a}$, is given by the expression,

$$
\kappa \nabla T(x)=-R \mathbf{J}^{2} \frac{x}{l_{a}}+\frac{R}{2} \mathbf{J}^{2}+K\left(T_{A}-T_{0}\right),
$$

which shows that $\nabla T(x)$ is enhanced with respect to $\left(T_{A}-T_{0}\right) / l_{a}$ for $0 \leq x \leq l_{a} / 2$ and is reduced for $l_{a} / 2 \leq x \leq l_{a}$. Since temperature is constant at the source and the sink, and $T(x)$ has a finite slope at $T_{0}$ and $T_{A}$, temperature is a continuous function with a kink at $T_{0}$ and $T_{A}$.

Thermal current. Substituting $\nabla T(x)$ given by Eq.(51) into Eq.(44) gives at the source $\left(x=0, T(0)=T_{0}\right)$,

$$
\left.\mathbf{J}_{Q}\right|_{x=0}=\alpha T_{0} \mathbf{J}-K\left(T_{A}-T_{0}\right)-\frac{R \mathbf{J}^{2}}{2} .
$$

The first term is the Peltier heat generated by the electrical current at point $x=0$ where the sample is attached to the reservoir, the second term is the heat flux diffusing from the source to the sink due to the temperature difference $\left(T_{A}-T_{0}\right)$, and the last term is $1 / 2$ of the Joule heat generated by constant current $\mathbf{J}$ in the sample of length $a$. Interestingly, one half of Joule heat generated in the sample is returned to the source. For $T_{A}>T_{0}$, the heat is taken out of the source, provided the Peltier heat overcomes the diffusion heat and $1 / 2$ of the Joule heat.

The thermal current arriving at the sink, is

$$
\left.\mathbf{J}_{Q}\right|_{x=a}=\alpha T_{A} \mathbf{J}-K\left(T_{A}-T_{0}\right)+\frac{R \mathbf{J}^{2}}{2},
$$


which shows that one half of Joule heat generated in the sample is taken away by the outgoing heat current and dumped into sink.

For given temperatures of the source and the sink, the heat current $\mathbf{J}_{Q}\left(T_{0}\right)$, defined by (52), is pumped out of the source in order to maintains the stationary state. The parasitic heat which enters the source, say, because of poor thermal insulation, is removed by the thermoelectric device which operates between $T_{0}$ and $T_{A}$ at current $\mathbf{J}$. The accompanying thermal field $T(x)$ is defined by Eq.(50). If the current is switched on when the source and sink temperature are equal, the heat current $\mathbf{J}_{Q}=\alpha \mathbf{J} T_{A}-R \mathbf{J}^{2} / 2$ starts flowing out of the source. As long as the parasitic heat which is entering the source is less than $\mathbf{J}_{Q}\left(T_{0}\right)$ (the heat current pumped out of the source in a stationary state), heat will be removed from the source and its temperature will drop. The stationary state is established at $T_{0}$, when the heat removed from the source matches the parasitic heat.

Figure of merit. For a given $T_{A}$ and $T_{0}$, the heat current pumped out of the source in a stationary state depends on $\mathbf{J}$, as shown by Eq.(52). The maximum cooling power at the source is obtained for $d J_{Q} / d \boldsymbol{J}=0$, which defines the optimal current,

$$
J_{o p t}=\frac{\alpha T_{0}}{R},
$$

and gives,

$$
J_{Q}^{\max }=\frac{\alpha^{2} T_{0}^{2}}{2 R}-K\left(T_{A}-T_{0}\right),
$$

But running the device with $J_{o p t}$ and removing the heat at the rate $J_{Q}^{\max }$ is not the most efficient mode of operation.

If the source is thermally isolated, the device will keep removing the heat and reducing $T_{0}$, as long as $\mathbf{J}_{Q}\left(T_{0}\right) \geq 0$. For given temperature of the sink $T_{A}$, the lowest $T_{0}$ (maximum of $\Delta T=T_{A}-T_{0}$ ) is reached when the thermoelectric reaches the state $\mathbf{J}_{Q}=0$, which gives,

$$
\left(T_{A}-T_{0}\right)=\frac{\alpha T_{0} \mathbf{J}}{K}-\frac{R \mathbf{J}^{2}}{2 K} .
$$

The maximum $\Delta T$ which one can achieve using a given thermoelectric material is obtained for $\mathbf{J}=j_{\text {opt }}$, such that

$$
\left(T_{A}-T_{0}\right)_{M a x}=\frac{1}{2} Z T_{0}^{2}
$$

where, $Z=\sigma \alpha^{2} / 2 \kappa$ is the figure-of-merit of a given thermoelectric.

Coefficient of performance. When the device operates as a fridge, the coefficient of performance is defined by the ratio of the heat taken from the source and the power supplied by the battery. This power has to maintain the heat flow from $T_{0}$ to $T_{A} \leq T_{0}$ and compensate the Joule losses and is given by the difference between the outgoing (source) and the incoming (sink) heat currents, $W=\left[\mathbf{J}_{Q}\right]_{x=0}-\left[\mathbf{J}_{Q}\right]_{x=a}$. Using Eqs. (52) and (53) we find,

$$
W=\alpha\left(T_{A}-T_{0}\right) \mathbf{J}+R \mathbf{J}^{2},
$$


so that the coefficient of performance is

$$
\Phi=\frac{q_{c}}{W}=\frac{\alpha T_{0} \mathbf{J}-K\left(T_{A}-T_{0}\right)-R \mathbf{J}^{2} / 2}{\alpha\left(T_{A}-T_{0}\right) \mathbf{J}+R \mathbf{J}^{2}} .
$$

The optimal performance follows from $d \Phi / d J=0$, which gives the optimal current

$$
J_{\Phi}=\frac{\alpha\left(T_{A}-T_{0}\right)}{R\left[\left(1+Z T_{M}\right)^{1 / 2}-1\right.},
$$

where $T_{M}=\left(T_{1}+T_{2}\right) / 2$. The best efficiency is,

$$
\Phi_{\max }=\frac{T_{0}}{T_{A}-T_{0}} \frac{\left(1+Z T_{M}\right)^{1 / 2}-T_{A} / T_{0}}{\left.1+Z T_{M}\right)^{1 / 2}+1} .
$$

$\Phi$ approaches the Carnot efficiency for $Z \rightarrow \infty$.

Efficiency coefficient. When the device is operating as a generator, the efficiency coefficient is defined by the ratio of the useful power of the external load, $W=\mathbf{J}^{2} R_{\text {Load }}$, and the heat taken from the source. (We now have, $T_{A}<T_{0}$.) The efficiency $\eta$ is defined as,

$$
\eta=\frac{W}{\mathbf{J}_{Q}}=\frac{\mathbf{J}^{2} R_{\text {Load }}}{\alpha T_{0} \mathbf{J}-K\left(T_{0}-T_{A}\right)-R \mathbf{J}^{2} / 2}
$$

The current is driven by the Seebeck voltage and given by $\mathbf{J}=\alpha\left(T_{0}-T_{A}\right) / R_{\text {Load }}$, where we neglected the resistance of the thermoelectric with respect to $R_{\text {Load }}$. As shown by Ioffe[6], maximizing with respect to $R_{\text {Load }} / R$ gives,

$$
\eta=\frac{\left(T_{0}-T_{A}\right)}{T_{0}} \frac{\sqrt{1+Z T_{M}}-1}{\sqrt{1+Z T_{M}}+T_{A} / T_{0}}
$$




\section{Hetero-structures}

Suppose we consider the device consisting of a thermoelectric B of length $b$ connected to the thermoelectric A of length $a$. The transport coefficients are $\sigma_{A}, \alpha_{A}, \kappa_{A}$, and $\sigma_{B}, \alpha_{B}, \kappa_{B}$, and the cross-sections are $A_{a}$ and $A_{b}$. The thermoelectric A extends from $x=0$ to $x=a$, where it connects to the sink at temperature $T_{A}$, and the thermoelectrics $B$ extends from $x=0$ to $x=-b$, where it connects to the source at $T_{B}$. The contact between the two thermoelectrics, at $x=0$, does not impede the heat and charge transport, and temperature at the contact is $T_{0}$.

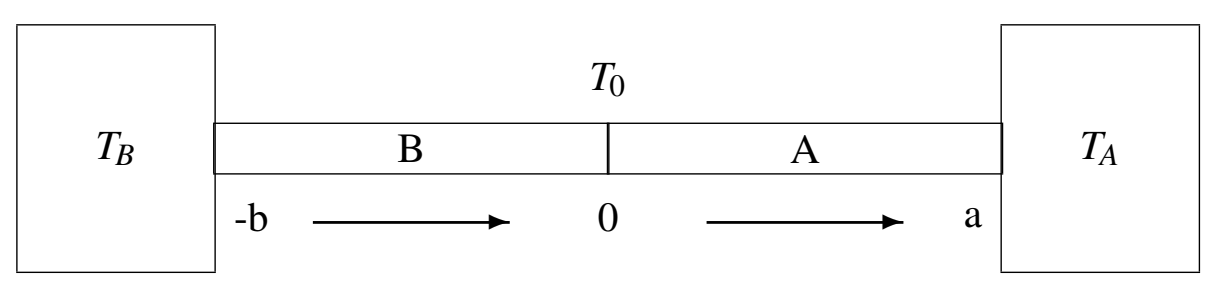

We consider the stationary state with constant electrical current, with the sink at temperature $T_{A}$, and the source at $T_{B}$. The heat $\mathbf{J}_{Q}^{B}$ is taken out of the source by the thermoelectric B and the heat $\mathbf{J}_{Q}^{A}$ dumped in the sink by A. The previous analysis shows that the cooling power of $\mathrm{B}$ depends on $T_{B}$ and $T_{0}$, and of $\mathrm{A}$ on $T_{A}$ and $T_{0}$. Assuming a stationary state and constant current we can find $T_{0}$, performing the analysis of the heat and charge transport in A and B in the same way as before. The boundary conditions are shown in the figure above.

The Domenicali equations for $\mathrm{A}$ and $\mathrm{B}$ are the same as before, and all the results are obtained by using Eqs. (47) to (51) with $\sigma_{A}, \alpha_{A}, \kappa_{A}$ for A, and by $\sigma_{B}, \kappa_{A}, \kappa_{B}$ for B, and by taking care of temperatures at the source, at the sink and at the boundary between $A$ and $\mathrm{B}$. For a general point $x \in(0, a)$ in $\mathrm{A}$ and $x \in(-b, 0)$ in $\mathrm{B}$ the solution can be written as,

$$
-\kappa_{A, B} \nabla T(x)=\frac{\mathbf{J}^{2}}{\sigma_{A, B}} x+F_{A, B},
$$

and

$$
-\kappa_{A, B} T(x)=\frac{\mathbf{J}^{2}}{\sigma_{A, B}} \frac{x^{2}}{2}+F_{A, B} x+G_{A, B},
$$

where the integration constants follow form the boundary condition for the thermal field. From Eq.(61) and $T(-b)=T_{B}, T(0)=T_{0}$, which holds in $\mathrm{B}$, we find $G_{B}=-\kappa_{B} T_{0}$, and

$$
F_{B}=-K_{B}\left(T_{0}-T_{B}\right)+R_{B} \mathbf{J}^{2} / 2
$$

while from $T(0)=T_{0}$, and $T(a)=T_{A}$, which holds in A, we find $G_{A}=-\kappa_{A} T_{0}$, and

$$
F_{A}=-K_{A}\left(T_{A}-T_{0}\right)-R_{A} \mathbf{J}^{2} / 2
$$


where $K_{A, B}$ and $R_{A, B}$ are the thermal conductance and the electrical resistance in $A$ and $B$, respectively. Note, the opposite sign of the Joule term in $F_{A}$ and $F_{B}$. However, the problem is not solved unless we specify $T_{0}$.

Temperature at the boundary follows from the condition that the heat current us continuous from A to B. Using Eq.(60) for $\nabla T(x)$ we calculate $\mathbf{J}_{Q}(x)=\alpha T \mathbf{J}-\kappa \nabla T(x)$, at $x=0$ in $\mathrm{B}$ and $\mathrm{A}$, which gives,

$$
\left.\mathbf{J}_{Q}^{B}\right|_{x=0}=\alpha_{B} T_{0} \mathbf{J}+F_{B}
$$

and

$$
\left.\mathbf{J}_{Q}^{A}\right|_{x=0}=\alpha_{A} T_{0} \mathbf{J}+F_{A},
$$

where $F_{A}$ and $F_{B}$ are the integration constants of Deminicalli equations in segments $\mathrm{A}$ and $\mathrm{B}$, respectively. Matching the heat currents gives an equation for $T_{0}$,

$$
\alpha_{B} T_{0} \mathbf{J}-K_{B}\left(T_{0}-T_{B}\right)+\frac{R_{B} \mathbf{J}^{2}}{2}=\alpha_{A} T_{0} \mathbf{J}-K_{A}\left(T_{A}-T_{0}\right)-\frac{R_{A} \mathbf{J}^{2}}{2} .
$$

which takes into account that $1 / 2$ of the Joule heat generated in $\mathrm{B}$ is transferred at the boundary to $A$, and that $1 / 2$ of the Joule heat generated in $A$ is returned to $B$. In the absence of electrical current, we have $K_{B}\left(T_{0}-T_{B}\right)=K_{A}\left(T_{A}-T_{0}\right)$, which gives

$$
T_{0}=\frac{K_{A} T_{A}+K_{B} T_{B}}{K_{A}+K_{B}}
$$

so that $\nabla T(x)$ has a break at $T_{0}$ unless $K_{A}=K_{B}$. If $\mathbf{J}=0$, the heat flows in the stationary state without any losses. But if $\mathbf{J} \neq 0$, the temperature profile is parabolic in each segment and the difference in the heat current entering at $B$ and leaving at $A$ is $R_{A}^{2} / 2+R_{B}^{2} / 2$.

Similarly, using the condition that the heat current leaving the source is equal to the heat current in the thermoelectric B at point $x=-b, \mathbf{J}_{Q}^{B}=\alpha_{B} T_{B} \mathbf{J}-\left.\kappa_{B} \nabla T\right|_{x=-b}$, we get an equation for $T_{B}$,

$$
\mathbf{J}_{Q 0}^{B}=\alpha_{B} T_{B} \mathbf{J}-K_{B}\left(T_{0}-T_{B}\right)-\frac{\mathbf{J}^{2} R_{B}}{2},
$$

if we specify the cooling rate at the source. Eqs.(64) and (65) provide a set of linear equations for $T_{B}$ and $T_{0}$.

We can now calculate the maximum temperature difference, $\Delta T$, the coefficient of performance and the efficiency coefficient, and find the currents which maximize these coefficients. We can then express these maximal values in terms of effective figures of merit $Z_{\Phi}^{*}$ and $Z_{\eta}^{*}$, which are defined by expressions (57) and (59). If $Z_{\Phi}^{*}$ and $Z_{\eta}^{*}$ are not too different, they can be used to specify the heterostructure in terms of material constants, and initial conditions.

$N$ segments. The generalization to $\mathrm{N}$ segments, shown in the figure below, is straightforward. In the $i$-th segment of length $l_{i}$, the solution of the Domenicali equation for $\nabla T(x)$ and $T(x)$ is given by Eqs.(61) and (60), where, say, $T_{B}, \alpha_{B}, \sigma_{B}, \kappa_{B}$ and $T_{0}$ are 
replaced by $T_{i-1}, \alpha_{i-1}, \sigma_{i-1}, \kappa_{i-1}$ and $T_{i}$, and $x$ is measured with respect to the initial point of the segment $x_{i-1}$. That is,

$$
-\kappa_{i} \nabla T(x)=\frac{\mathbf{J}^{2}}{\sigma_{i}} x+F_{i} \quad \text { for } x_{i-1} \leq x \leq i
$$

and

$$
-\kappa_{i} T(x)=\frac{\mathbf{J}^{2}}{\sigma_{i}} \frac{x^{2}}{2}+F_{i} x+G_{i} \quad \text { for } x_{i-1} \leq x \leq i,
$$

and the corresponding integration constants are $G_{i}=-T_{i}$ and

$$
F_{i}=-K_{i}\left(T_{i}-T_{i-1}\right)+R_{i} \mathbf{J}^{2} / 2,
$$

The solution has to satisfy the condition that temperature, electrochemical potential, and normal components of heat current and electrical current, are continuous at every point, which gives,

$$
\lim _{\delta \rightarrow 0}\left[\alpha_{i-1} T_{i-1} \mathbf{J}-\left.\kappa_{i-1} \nabla T_{i-1}\right|_{x_{i}-\delta}=\lim _{\delta \rightarrow 0}\left[\alpha_{i} T_{i} \mathbf{J}-\kappa_{i} \nabla T_{i}\right]_{x_{i}+\delta}\right.
$$

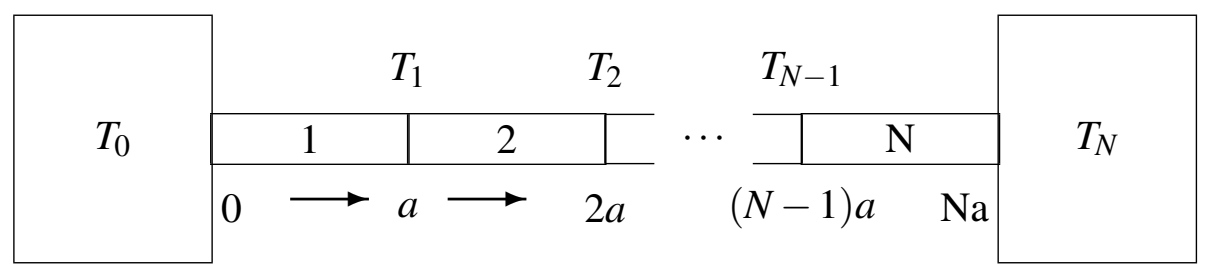

The boundary conditions at $x=0, \ldots, x=N(a-$ 1), provide $N$ equations for temperature field defined at points $\mathbf{t}=\left\{T_{0}, \ldots, T_{N-1}\right\}$. Assuming we know $T_{N}$ and $\mathbf{J}_{Q}^{0}$, Eq.(68) give the set of equations,

$$
\begin{aligned}
& -\alpha_{1} T_{0} \mathbf{J}+K_{1}\left(T_{1}-T_{0}\right)=-\left(\mathbf{J}_{Q}^{0}+\frac{\mathbf{J}^{2}}{2} R_{1}^{2}\right) \\
& \alpha_{1} T_{1} \mathbf{J}-K_{1}\left(T_{1}-T_{0}\right)-\alpha_{2} T_{1} \mathbf{J}+K_{2}\left(T_{2}-T_{1}\right)=-\frac{\mathbf{J}^{2}}{2}\left(R_{1}^{2}+R_{2}^{2}\right) \\
& \alpha_{2} T_{2} \mathbf{J}-K_{2}\left(T_{2}-T_{1}\right)-\alpha_{3} T_{2} \mathbf{J}+K_{3}\left(T_{3}-T_{2}\right)=-\frac{\mathbf{J}^{2}}{2}\left(R_{2}^{2}+R_{3}^{2}\right) \\
& \alpha_{i-1} T_{i-1} \mathbf{J}-K_{i-1}\left(T_{i-1}-T_{i-2}\right)-\alpha_{i} T_{i-1} \mathbf{J}+K_{i}\left(T_{i}-T_{i-1}\right)=-\frac{\mathbf{J}^{2}}{2}\left(R_{i-1}^{2}+R_{i}^{2}\right) \\
& \alpha_{N-1} T_{N-1} \mathbf{J}-K_{N}\left(T_{N-1}-T_{N-2}\right)-\alpha_{N} T_{N-1} \mathbf{J}+K_{N}\left(T_{N}-T_{N-1}\right)=-\frac{\mathbf{J}^{2}}{2}\left(R_{N-1}^{2}+R_{N}^{2}\right)
\end{aligned}
$$

which is an array with elements on the main diagonal and two principal sub-diagonals,

$-\left(\alpha_{1} \mathbf{J}-K_{1}\right) T_{0}+K_{1} T_{1}$

Correlated thermoelectrics

$$
=-\left(\mathbf{J}_{Q}^{0}+\frac{\mathbf{J}^{2}}{2} R_{1}^{2}\right)
$$




$$
\begin{aligned}
& K_{1} T_{0}+\left[\left(\alpha_{1}-\alpha_{2}\right) \mathbf{J}-\left(K_{1}+K_{2}\right)\right] T_{1}+K_{2} T_{2} \quad=-\frac{\mathbf{J}^{2}}{2}\left(R_{1}^{2}+R_{2}^{2}\right) \\
& K_{2} T_{1}+\left[\left(\alpha_{2}-\alpha_{3}\right) \mathbf{J}-\left(K_{2}+K_{3}\right)\right] T_{2}+K_{3} T_{3}=-\frac{\mathbf{J}^{2}}{2}\left(R_{2}^{2}+R_{3}^{2}\right) \\
& \text { ․ } \quad \ldots \quad \ldots \\
& K_{i-1} T_{i-2}+\left[\left(\alpha_{i-1}-\alpha_{i}\right) \mathbf{J}-\left(K_{i-1}+K_{i}\right)\right] T_{i-1}+K_{i} T_{i}=-\frac{\mathbf{J}^{2}}{2}\left(R_{i-1}^{2}+R_{i}^{2}\right) \\
& K_{N-1} T_{N-2}+\left[\left(\alpha_{N-1}-\alpha_{N}\right) \mathbf{J}-\left(K_{N-1}+K_{N}\right)\right] T_{N-1}=-\frac{\mathbf{J}^{2}}{2}\left(R_{N-1}^{2}+R_{N}^{2}\right)-K_{N} T_{N}
\end{aligned}
$$

or in the matrix form

$$
\mathbf{M t}=-\mathbf{c}-\frac{\mathbf{J}^{2}}{2}(\mathbf{r}+\mathbf{R})
$$

where $\mathbf{c}=\left\{\mathbf{q}_{\mathbf{0}} \mathbf{J}^{2} / 2,0, \ldots, 0, K_{N} T_{N}\right\}, \mathbf{r}=\left\{R_{0}^{2}, R_{1}^{2}, \ldots, R_{N-1}^{2}\right\}$, and $\mathbf{R}=\left\{R_{1}^{2}, R_{2}^{2}, \ldots, R_{N}^{2}\right\}$.

The matrix elements of $\mathbf{M}$ are defined as $M_{11}=-\alpha_{1} \mathbf{J}+K_{1}$, and

$M_{i j}=\left[\left(\alpha_{i-1}-\alpha_{i}\right) \mathbf{J}-\left(K_{i-1}+K_{i}\right)\right] \delta_{i j}+K_{i} \delta_{i, j-1}+K_{i-1} \delta_{i, j+1}$ for $i, j \geq 1$.

Provided $\operatorname{det} \mathbf{M} \neq 0$ we can find the solution

$$
\mathbf{t}=\mathbf{M}^{-\mathbf{1}}\left[-\mathbf{c}-\frac{\mathbf{J}^{2}}{\mathbf{2}}(\mathbf{r}+\mathbf{R})\right]
$$

for a given set of material constants, $\mathbf{A}=\left\{\alpha_{1}, \ldots, \alpha_{N}\right\}, \mathbf{K}=\left\{\kappa_{1}, \ldots, \kappa_{N}\right\}, \mathbf{R}$, and for a given value of $T_{N}, \mathbf{J}$, and the heat current at the source $\mathbf{q}_{\mathbf{0}}$.

Alternatively, we can rewrite the first equation as

$$
\left.\mathbf{J}_{Q}^{0}+K_{1} T_{1}=-\frac{\mathbf{J}^{2}}{2} R_{1}^{2}\right)+-\left(\alpha_{1} \mathbf{J}-K_{1}\right) T_{0}
$$

and invert new matrix matrix $\mathbf{M}^{\prime}$ to find $\mathbf{J}_{Q}^{0}$ and $\left\{T_{1}, \ldots, T_{N-1}\right\}$ in terms of $T_{0}$ and $T_{N}$. If the material-specific constants are strongly temperature dependent, the calculations have to be performed self-consistently. That is, we start with some initial value of $\mathbf{A}, \mathbf{K}$, and $\mathbf{R}$, calculated for the linear temperature dependence of $T(x)$ between $T_{0}$ and $T_{N}$. We then solve Eq.(70) to find $T(x), \nabla T(x)$ and the heat current in each segment. Then we recalculate $\mathbf{A}, \mathbf{K}$, and $\mathbf{R}$, and iterate unless the result converges.

The same procedure has to be performed for the Seebeck effect, when $\mathbf{J}=0$ and we know $\Delta T=T_{0}-T_{a}$. Eventually, we obtain the electrical field $\mathbf{E}(x)=\alpha(x) \nabla T(x)$, evaluate $\Delta V=\int_{0}^{l} d x \mathbf{E}(x)$, and define the effective Seebeck coefficient of the heterostructure at $\left.\left(T_{0}+T\right) N\right) / 2$ as $S^{*}=\Delta V / \Delta T$.

\section{LINEAR RESPONSE THEORY}

The coefficients appearing in the transport equations can either be treated as phenomenological parameters taken from the experiment on a given sample or calculated for a 
microscopic model that we use to describe this sample. Here, we follow Luttinger's approach[2] to calculate the transport coefficients for a given Hamiltonian

The macroscopic values of the charge and heat currents densities are defined as the statistical averages

$$
\begin{gathered}
\mathbf{J}=\operatorname{Tr}\left\{\rho_{\phi} \hat{\mathbf{j}}\right\}, \\
\mathbf{J}_{u}=\operatorname{Tr}\left\{\rho_{\phi} \hat{\mathbf{j}}_{u}\right\},
\end{gathered}
$$

where $\hat{\mathbf{j}}$ and $\hat{\mathbf{j}}_{u}$ are the charge current density and the energy current density operators, respectively. The Hamiltonian is

$$
\hat{H}_{\phi}=\hat{H}+\Delta H_{\phi}(t)=\hat{H}+e^{\omega t} \hat{F},
$$

where $\hat{H}$ defines the dynamics of interacting particles in the absence of the external field and $\Delta H_{\phi}(t)=e^{\omega t} \hat{F}$ is the perturbation. The operator

$$
\hat{F}=\int d^{3} x \phi(x) e \hat{n}(x)
$$

provides the coupling of the charge density $e \hat{n}(x)$ to the external potential which is gradually turned on, as described by the exponential factor $e^{\omega t}$. The coupling is absent at $t=-\infty$ and is fully turned on at $t=0$. Note, a completely different behavior is obtained for 'slow' and 'rapid' switching-on of the perturbation. (The time-scale for the 'slow' and 'rapid' perturbation is set by the characteristic time-scales of the unperturbed system.) In the 'slow' case, the electron wavefunctions adjust to the new potential and the stationary state corresponds to thermal equilibrium, where no currents flow. In the 'rapid' case, the wave functions are not modified by the perturbation; the additional potential generates the currents which flow so as to establish the equilibrium. For a potential characterized by a single spatial Fourier component $\phi_{q}$, the switching is rapid if $q / \omega \rightarrow 0$ which describes a uniform system perturbed by a time-dependent potential (the limit $q \rightarrow 0$ is taken before $\omega \rightarrow 0$ ). The switching is slow if $\omega / q \rightarrow 0$, which describes a static perturbation of a spatially modulated system without any currents (the limit $\omega \rightarrow 0$ is taken before $q \rightarrow 0$ ).

The density matrix $\rho_{\phi}$ is defined by the equation

$$
i \frac{\partial \rho_{\phi}}{\partial t}=\left[\hat{H}_{\phi}, \rho_{\phi}\right]
$$

and the initial condition $\rho_{\phi}(-\infty)=\rho_{0}$. The unperturbed density matrix is $\rho_{0}$ and we want the lowest order correction to $\rho_{0}$ due to $\Delta H(t)$. Writing

$$
\rho_{\phi}(t)=\rho_{0}+f(t)
$$

we obtain from the equation of motion

$$
i \frac{\partial f(t)}{\partial t}=\left[\hat{H}, \rho_{0}\right]+[\hat{H}, f]+\left[\Delta \hat{H}_{\phi}, \rho_{0}\right]+\left[\Delta \hat{H}_{\phi}, f\right]
$$


which has to be solved for the boundary condition $f(-\infty)=0$. Using $\left[\hat{H}, \rho_{0}\right]=0$ and neglecting the non-linear term gives

$$
i \frac{\partial f(t)}{\partial t}-[\hat{H}, f]=\left[\Delta \hat{H}_{\phi}, \rho_{0}\right] .
$$

The identity

$$
i \frac{\partial f(t)}{\partial t}-[\hat{H}, f]=e^{-H t} i\left\{\frac{\partial}{\partial t} e^{H t} f(t) e^{-H t}\right\} e^{H t}
$$

allows us to write

$$
i \frac{\partial}{\partial t} e^{H t} f(t) e^{-H t}=e^{H t}\left[\Delta \hat{H}_{\phi}, \rho_{0}\right] e^{-H t}=\left[\Delta \hat{H}_{\phi}(t), \rho_{0}\right]
$$

such that

$$
e^{H t} f(t) e^{-H t}=f(-\infty)-i \int_{-\infty}^{t} d t^{\prime}\left[\Delta \hat{H}_{\phi}\left(t^{\prime}\right), \rho_{0}\right]
$$

Using $f(-\infty)=0$ obtains and

$$
f(t)=-i \int_{-\infty}^{t} d t^{\prime}\left[\Delta \hat{H}_{\phi}\left(t^{\prime}-t\right), \rho_{0}\right]=-i \int_{0}^{\infty} d t^{\prime} e^{-\omega t^{\prime}}\left[F\left(-t^{\prime}\right), \rho_{0}\right] .
$$

In the last equation we shifted the integration variable by $t$ and used $F(z)=$ $e^{i H z} F(t) e^{-i H z}$ for $z$ in the complex plane. We now show that $f(t)$ is proportional to the gradient of the external potential. The identity

$$
\left[F\left(-t^{\prime}\right), \rho_{0}\right]=-i \rho_{0} \int_{0}^{\beta} d \beta^{\prime} \frac{\partial F\left(-t^{\prime}-i \beta^{\prime}\right)}{\partial t^{\prime}}
$$

gives

$$
f=-\rho_{0} \int_{0}^{\infty} d t^{\prime} e^{-\omega t^{\prime}} \int_{0}^{\beta} d \beta^{\prime} \frac{\partial F\left(-t^{\prime}-i \beta^{\prime}\right)}{\partial t}
$$

and form the definition of the operator $\hat{F}$ and the continuity equation for the number density operator we find

$$
\frac{\partial F(-t-i \beta)}{\partial t}=\int d^{3} x \phi(x) e^{\beta H} \frac{\partial \hat{n}(-t)}{\partial t} e^{-\beta H}=\int d^{3} x \phi(x) \nabla \cdot \hat{\mathbf{j}}(x,-t-i \beta) .
$$

Finally, the integration by parts gives

$$
\frac{\partial F(-t-i \beta)}{\partial t}=-\int d^{3} x^{\prime} \hat{\mathbf{j}}\left(x^{\prime},-t-i \beta\right) \cdot \mathbf{E}\left(x^{\prime}\right),
$$

where $\mathbf{E}(x)=-\nabla \phi(x)$. Thus, the lowest order correction to $\rho_{\phi}(t)$ is proportional to the applied field and given by

$$
f=+\rho_{0} \sum_{\gamma} \int d^{3} x^{\prime} \int_{0}^{\infty} d t^{\prime} e^{-\omega t^{\prime}} \int_{0}^{\beta} d \beta^{\prime} \mathbf{E}_{\gamma}\left(x^{\prime}\right) \hat{\mathbf{j}}_{\gamma}\left(x^{\prime},-t^{\prime}-i \beta^{\prime}\right) .
$$


Since no current flows for $\mathbf{E}(x)=0$, the current density induced by the field is

$$
\mathbf{J}_{\alpha}(x)=\operatorname{Tr}\left\{f \hat{\mathbf{j}}_{\alpha}\right\}=\sum_{\gamma} \int d^{3} x^{\prime} \mathbf{E}_{\gamma}\left(x^{\prime}\right) \int_{0}^{\infty} d t^{\prime} e^{-\omega t^{\prime}} \int_{0}^{\beta} d \beta^{\prime}<\hat{\mathbf{j}}_{\gamma}\left(x^{\prime},-t^{\prime}-i \beta^{\prime}\right) \hat{\mathbf{j}}_{\alpha}(x)>_{0},
$$

where $\langle\cdots\rangle_{0}$ denotes the thermodynamic average with respect to $\rho_{0}$ and the $\alpha, \gamma$ label the spatial directions.

The above expression gives the thermodynamic average of the current density at point $\mathbf{x}$ induced by a spatially varying electrical field. To compare with the phenomenological equations discussed in the first part of these lecure notes we shoud make an additional averaging of the current given by Eq. (85) over a macroscopic region aroud $\mathbf{x}$. Thus, we introduce the Fourier transform of the current density,

$$
\mathbf{J}_{\alpha}(q)=\frac{1}{V} \int d^{3} x e^{i \mathbf{q} \cdot \mathbf{x}} \mathbf{J}_{\alpha}(x)
$$

denote by $\hat{\mathbf{j}}_{\gamma}(q)$ the Fourier component of current density operator, and consider the external potential with a single Fourier component,

$$
\phi(x)=\phi_{q} e^{i q x} .
$$

The electrical field is

$$
\mathbf{E}_{\gamma}(x)=\mathbf{E}_{\gamma}(q) e^{i \mathbf{q} \cdot \mathbf{x}}
$$

where

$$
\mathbf{E}_{\gamma}(q)=-i q_{\gamma} \phi_{q}
$$

The only non-vanishing Fourier component of the macroscopic current density is then

$$
\mathbf{J}_{\alpha}(q)=\sum_{\gamma} \mathbf{E}_{\gamma}(q) \int_{0}^{\infty} d t^{\prime} e^{-\omega t^{\prime}} \int_{0}^{\beta} d \beta^{\prime}<\hat{\mathbf{j}}_{\gamma}\left(-q,-t^{\prime}-i \beta^{\prime}\right) \hat{\mathbf{j}}_{\alpha}(q)>_{0}
$$

and to compare with the phenomenological expression for the current density we should now take the limit $q, \omega \rightarrow 0$. If the limit $\omega \rightarrow 0$ is taken with finite $q$, the above expression describes the electrons moving in a static periodic potential and $\mathbf{J}_{\alpha}(q)$ should vanish. In general, the correlator in Eq.(90) does not have to vanish in that limit, which means that the above result is unphysical in that limit. The reason is that the physical zero-current state corresponds to a balance between the electrical force, which is due to the potential gradient, and the diffusion force, which is due to the concentration gradient. Since the diffusion force cannot be included in the Hamiltonian, Eq.(90) does not give the correct result, if the limit $\omega \rightarrow 0$ is taken before $q \rightarrow 0$.) However, if $q \rightarrow 0$ is taken before $\omega \rightarrow 0$, we find the current that is flowing in response to the external field that has been gradually turned on. The average current obtained by the linear-response theory in such a way should coincide with the phenomenological current written for the sample without the thermal force and the diffusion force. Writing

$$
\mathbf{J}_{\alpha}=\lim _{q \rightarrow 0} \mathbf{J}_{\alpha}(q)=\lim _{q \rightarrow 0} \sum_{\gamma} \sigma_{\gamma \alpha} \mathbf{E}_{\gamma}(q),
$$


we define the static conductivity by the expression

$$
\sigma_{\gamma \alpha}=\lim _{\omega \rightarrow 0} \int_{0}^{\infty} d t^{\prime} e^{-\omega t^{\prime}} \int_{0}^{\beta} d \beta^{\prime}<\hat{\mathbf{j}}_{0 \gamma}\left(-t^{\prime}-i \beta^{\prime}\right) \hat{\mathbf{j}}_{0 \alpha}>_{0}
$$

where $\mathbf{J}_{\alpha}$ and $\hat{\mathbf{j}}_{0 \gamma}$ denote the $q=0$ Fourier components of $\mathbf{J}_{\alpha}(q)$ and $\hat{\mathbf{j}}_{\gamma}(q)$, respectively. Equating $\mathbf{J}_{\alpha}$ with the macroscopic current density in Eq. (19) or (21), we relate the phenomenological transport coefficient $L_{11}$ to the correlation function for the static conductivity $\sigma_{\gamma \alpha}$.

In real space, the static conductivity is defined by the expression

$$
\mathbf{J}_{\alpha}(x)=\sum_{\gamma} \int d^{3} x^{\prime} \mathbf{E}_{\gamma}\left(x^{\prime}\right) \sigma_{\gamma, \alpha}\left(x, x^{\prime}\right)
$$

where

$$
\sigma_{\gamma, \alpha}\left(x, x^{\prime}\right)=\lim _{\omega \rightarrow 0} \int_{0}^{\infty} d t^{\prime} e^{-\omega t^{\prime}} \int_{0}^{\beta} d \beta^{\prime}<\hat{\mathbf{j}}_{\gamma}\left(x^{\prime},-t^{\prime}-i \beta^{\prime}\right) \hat{\mathbf{j}}_{\alpha}(x)>_{0}
$$

This expression should be used for inhomogeneous materials. In translationally invariant systems, the Fourier transform of (94) gives the same results as obtaioned before.

The uniform static conductivity given by expression Eq. (92) agrees with the usual Kubo formula obtained by calculating the $\omega \rightarrow 0$ limit of the dynamical conductivity. In the basis which diagonalizes $H$, we have

$$
\begin{aligned}
<n\left|\hat{\mathbf{j}}_{0 \gamma}(-t-i \beta)\right| m> & =<n\left|e^{i H(-t-i \beta)} \hat{\mathbf{j}}_{0 \gamma}(0) e^{-H(-t-i \beta)}\right| m> \\
& =e^{-i\left(E_{n}-E_{m}\right)(-t-i \beta)}<n\left|\hat{\mathbf{j}}_{0 \gamma}(0)\right| m>
\end{aligned}
$$

Then, taking the trace with respect to $\rho_{0}$ we obtain from Eq. (92) the static conductivity

$$
\sigma_{\gamma \alpha}=\operatorname{Re}\left\{\sum_{m n} e^{-E_{m} \beta}<n\left|\hat{\mathbf{j}}_{0 \gamma}\right| m><m\left|\hat{\mathbf{j}}_{0 \alpha}\right| n>\right\} \int_{0}^{\infty} d t e^{i t\left(E_{n}-E_{m}+i \omega\right)} \int_{0}^{\beta} d \beta e^{\left.\left(E_{n}-E_{m}\right) \beta\right)} .
$$

The limit

$$
\lim _{\omega \rightarrow 0} \operatorname{Re} \int_{0}^{\infty} d t e^{i t\left(E_{n}-E_{m}+i \omega\right)}=\pi \delta\left(E_{n}-E_{m}\right)
$$

and

$$
\delta\left(E_{n}-E_{m}\right) \int_{0}^{\beta} d \beta e^{\left.\left(E_{n}-E_{m}\right) \beta\right)}=\beta \delta\left(E_{n}-E_{m}\right)
$$

provide the uniform conductivity matrix

$$
\sigma_{\gamma \alpha}=\pi \beta \sum_{m n} e^{-E_{m} \beta}<n\left|\hat{\mathbf{j}}_{0 \gamma}\right| m><m\left|\hat{\mathbf{j}}_{0 \alpha}\right| n>\beta \delta\left(E_{n}-E_{m}\right),
$$

that coincides with the spectral representation obtained from the Kubo expression for the dynamical conductivity. 
In Kubo formalism, the transport coefficients appearing in the phenomenological equations are expressed in terms of appropriate zero-frequency "polarization operators"[4]. For the coeficients in Eq. (21) we have[4],

$$
\bar{L}_{11}^{\gamma \alpha}=T \sigma_{\gamma \alpha}
$$

such that

$$
\begin{aligned}
\bar{L}_{11}^{\gamma \alpha} & =\lim _{v \rightarrow 0} \operatorname{Re} \frac{i}{v} \mathscr{L}_{11}^{\gamma \alpha}(v), \\
\mathscr{L}_{11}^{\gamma \alpha}\left(i v_{l}\right), & =\pi T \int_{0}^{\beta} d \tau e^{i v_{l} \tau}\left\langle T_{\tau} j_{0 \alpha}^{\dagger}(\tau) j_{0 \gamma}(0)\right\rangle,
\end{aligned}
$$

where $v_{l}=2 \pi T l$ is the Bosonic Matsubara frequency, the $\tau$-dependence of the operator is with respect to the full Hamiltonian in Eq. (183), and we must analytically continue $\mathscr{L}_{11}^{\gamma \alpha}\left(i v_{l}\right)$ to the real axis $\mathscr{L}_{11}^{\gamma \alpha}(v)$ before taking the limit $v \rightarrow 0$.

The derivation of the coefficient $\bar{L}_{21}^{\gamma \alpha}$ that gives the energy current density induced by an applied electrical field, proceeds in the same way and one easily finds[4],

$$
\begin{aligned}
\bar{L}_{21}^{\gamma \alpha} & =\lim _{v \rightarrow 0} \operatorname{Re} \frac{i}{v} \mathscr{L}_{12}^{\gamma \alpha}(v), \\
\mathscr{L}_{21}^{\gamma \alpha}\left(i v_{l}\right) & =\pi T \int_{0}^{\beta} d \tau e^{i v_{l} \tau}\left\langle T_{\tau} j_{0 Q \alpha}^{\dagger}(\tau) j_{0 \beta}(0)\right\rangle .
\end{aligned}
$$

However, the derivation of $\bar{L}_{12}^{\gamma \alpha}$ and $\bar{L}_{22}^{\gamma \alpha}$ coefficients that describe the currents induced by the thermal gradient is more complicated, because the 'thermal force' (like the 'diffusion force') does not appear in the Hamiltonian. The details are given in Ref. [2] and we just quote the results,

$$
\begin{aligned}
\bar{L}_{22}^{\gamma \alpha} & =\lim _{v \rightarrow 0} \operatorname{Re} \frac{i}{v} \mathscr{L}_{22}^{\gamma \alpha}(v), \\
\mathscr{L}_{22}^{\gamma \alpha}\left(i v_{l}\right) & =\pi T \int_{0}^{\beta} d \tau e^{i v_{l} \tau}\left\langle T_{\tau} j_{0 Q \alpha}^{\dagger}(\tau) j_{0 Q \beta}(0)\right\rangle .
\end{aligned}
$$

and $\bar{L}_{12}^{\gamma \alpha}=\bar{L}_{21}^{\gamma \alpha}$, in agreement with the Onsager relation. In all of these equations, the subscripts $\alpha$ and $\beta$ denote the respective spatial index of the current vectors and subscript 0 denotes the $q \rightarrow 0$ Fourier component.

In terms of transport coefficients $\bar{L}_{i j}^{\gamma \alpha}$ we then have

$$
\alpha=-\frac{k_{B}}{|e| T} \frac{\bar{L}_{12}^{\gamma \alpha}}{\bar{L}_{11}^{\gamma \alpha}},
$$

for the thermopower and

$$
\kappa=\frac{k_{B}^{2}}{T^{2}}\left[\bar{L}_{22}^{\gamma \alpha}-\frac{\bar{L}_{12}^{\gamma \alpha} \bar{L}_{21}^{\gamma \alpha}}{\bar{L}_{11}^{\gamma \alpha}}\right] .
$$

for the thermal conductivity. 


\section{CURRENT OPERATORS}

The linear response theory expresses the transport coefficients in terms of the thermal averages of the current operators. In this Section we calculate these operators for a given Hamiltonian using the conservation laws for the local charge and energy density. That is, we define the divergence of the current densities by the rate of change of the corresponding densities. The current density operators obtained in such a way give the macroscopic current densities that satisfy the continuity equations (EOC),

$$
\frac{\partial<e \hat{n}(\mathbf{x}, t)>}{\partial t}=-<\operatorname{div} \hat{\mathbf{j}}(\mathbf{x}, t)>
$$

and

$$
\frac{\partial<\hat{h}_{\phi}(\mathbf{x}, t)>}{\partial t}=-<\operatorname{div} \hat{\mathbf{j}}_{E}^{\phi}(\mathbf{x}, t)>.
$$

Here, $<\hat{n}(\mathbf{x} ; t)>=\operatorname{Tr}\left\{\rho_{\phi} \hat{n}(\mathbf{x})\right\},<\hat{h}_{\phi}(\mathbf{x} ; t)>=\operatorname{Tr}\left\{\rho_{\phi} \hat{h}_{\phi}(\mathbf{x})\right\}$ and the averaging is with respect to the Hamiltonian $\hat{H}_{\phi}=\int d^{3} x \hat{h}_{\phi}(\mathbf{x}, t)$. The energy density operator is $\hat{h}_{\phi}(\mathbf{x})=h(\mathbf{x})+\Delta \hat{h}_{\phi}(\mathbf{x})$, and the perturbation is $\Delta \hat{h}_{\phi}(\mathbf{x})=\phi(x) e \hat{n}(x)$.

Charge current. The equation of motion for the density matrix Eqs. (73) and the charge conservation expressed by (104) give

$$
\frac{\partial<\hat{n}(\mathbf{x}, t)>}{\partial t}=\operatorname{Tr}\left\{\frac{\partial \rho_{\phi}}{\partial t} \hat{n}(\mathbf{x})\right\}=\left\langle i\left[\hat{H}_{\phi}, \hat{n}(\mathbf{x})\right]\right\rangle,
$$

where the last equation follows from the cyclic invariance of the trace. Thus, the current density operator defined by the equation

$$
-\operatorname{div} \hat{\mathbf{j}}_{\phi}(x, t)=i\left[\hat{H}_{\phi}, e \hat{n}(\mathbf{x})\right]
$$

gives the macroscopic current density which satisfies the EOC. In the steady-state, reached at $t=0$, the flow is divergence-less: $\operatorname{div}\left\langle\hat{\mathbf{j}}_{\phi}(x, t)\right\rangle=0$.

The $q=0$ Fourier component of the current density operator $\hat{\mathbf{j}}_{\phi}=\int d^{3} x \hat{\mathbf{j}}_{\phi}(x, t)$ is obtained by commuting the Hamiltonian with the charge polarization operator

$$
\mathbf{P}_{c}(t)=e \int d^{3} x \mathbf{x} \hat{n}(x, t)
$$

We find, using (108), (107) and integrating by parts, the result

$$
i\left[\hat{H}_{\phi}, \mathbf{P}_{c}\right]=\int d^{3} x \mathbf{x} i\left[\hat{H}_{\phi}, e \hat{n}(\mathbf{x})\right]=-\int d^{3} x \mathbf{x} \operatorname{div} \hat{\mathbf{j}}_{\phi}(x, t)=\int d^{3} x \hat{\mathbf{j}}_{\phi}(x, t)=\hat{\mathbf{j}}_{\phi} .
$$

The current density operator is defined by the commutator

$$
\hat{\mathbf{j}}_{\phi}(x, t)=i\left[\hat{H}_{\phi}, \mathbf{x} e \hat{n}(\mathbf{x})\right] \text {. }
$$

For scalar gauge, such that $\hat{H}_{\phi}=\hat{H}+e \int d^{3} x \phi(x) \hat{n}(x)$, the current density operator defined by Eq. (110) is unaffected by the potential term of the Hamiltonian and we have $\hat{\mathbf{j}}_{\phi}=\hat{\mathbf{j}}$. 
Energy current. The energy current density operator follows in the same way. The local energy conservation gives

$$
\frac{\partial<\hat{h}_{\phi}(\mathbf{x}, t)>}{\partial t}=\operatorname{Tr}\left\{\frac{\partial \rho_{\phi}}{\partial t} \hat{h}_{\phi}(\mathbf{x})\right\}=<i\left[\hat{H}_{\phi}, \hat{h}_{\phi}(\mathbf{x})\right]>
$$

which we use to define the divergence of the energy current density

$$
-\operatorname{div} \hat{\mathbf{j}}_{u}(x, t)=i\left[\hat{H}_{\phi}, \hat{h}_{\phi}(x)\right]
$$

The $q=0$ Fourier component of the energy current density operator $\hat{\mathbf{j}}_{u}=\int d^{3} x \hat{\mathbf{j}}_{u}(x, t)$ is obtained by commuting the Hamiltonian with the energy polarization operator

$$
\mathbf{P}_{u}(t)=\int d^{3} x \mathbf{x} \hat{h}_{\phi}(x)
$$

This gives

$$
i\left[\hat{H}_{\phi}, \mathbf{P}_{u}\right]=\int d^{3} x \mathbf{x} i\left[\hat{H}_{\phi}, \hat{h}_{\phi}(\mathbf{x})\right]=-\int d^{3} x \mathbf{x} \operatorname{div} \hat{\mathbf{j}}_{u}(x, t)=\int d^{3} x \hat{\mathbf{j}}_{u}(x, t)=\hat{\mathbf{j}}_{u}
$$

and we take the commutator

$$
\hat{\mathbf{j}}_{u}(x, t)=i\left[\hat{H}_{\phi}, \mathbf{x} \hat{h}_{\phi}(x)\right]
$$

as the definition of the energy current density operator. This operator has contributions coming from

$$
i\left[\hat{H}_{\phi}, \hat{h}_{\phi}(\mathbf{x})\right]=i\left[\hat{H}_{\phi}, \hat{h}(\mathbf{x})\right]+i\left[\hat{H}, \Delta h_{\phi}(\mathbf{x})\right]+i\left[\Delta H, \Delta h_{\phi}(\mathbf{x})\right] .
$$

The last term in (116) vanishes and the contribution of the middle term to the total energy current is

$$
\hat{\mathbf{j}}_{u}^{\phi}=i\left[\hat{H}, \mathbf{P}_{u}^{\phi}(t)\right]
$$

where we introduced

$$
\mathbf{P}_{u}^{\phi}(t)=e \int d^{3} x \mathbf{x} \hat{\phi}(x) n(x, t)
$$

Eqs. (108) and (110) give

$$
\hat{\mathbf{j}}_{u}^{\phi}=\int d^{3} x e \phi(x) i[\hat{H}, \hat{n}(\mathbf{x})]=-\int d^{3} x \mathbf{x} \phi(x) \operatorname{div} \hat{\mathbf{j}}(x, t) .
$$

which becomes by partial integration

$$
\hat{\mathbf{j}}_{u}^{\phi}=\int d^{3} x \phi(x) \hat{\mathbf{j}}(x, t),
$$

where we neglected $\nabla \phi$ with respect to $\phi$. Thus, $\hat{\mathbf{j}}_{u}^{\phi}(x)=\phi(x) \hat{\mathbf{j}}(x)$. Performing similar analysis for the first term in Eq. (116), and neglecting again the gradient terms, gives

$$
-\operatorname{div} \hat{\mathbf{j}}_{E}^{\phi} \simeq-\operatorname{div} \hat{\mathbf{j}}_{E}
$$


where

$$
\hat{\mathbf{j}}_{E}=i\left[\hat{H}, \mathbf{P}_{E}\right],
$$

defines the energy current in the absence of the field and

$$
\mathbf{P}_{E}(t)=\int d^{3} x \mathbf{x} \hat{h}(x, t)
$$

is the field-free energy polarization operator. The full energy current density operator which satisfies the continuity equation is

$$
\hat{\mathbf{j}}_{u}(x)=\hat{\mathbf{j}}_{E}(x)+\phi(x) \hat{\mathbf{j}}(x) .
$$

In a stationary state, the rate of change of the total energy density around point $\mathbf{x}$ is zero, such that $\dot{u}=-\operatorname{div}<\hat{\mathbf{j}}_{u}(\mathbf{x})>=0$. However, the internal energy is not conserved and $-\operatorname{div}<\hat{\mathbf{j}}_{E}(\mathbf{x})>\neq 0$. None-the-less $\hat{\mathbf{j}}_{E}(\mathbf{x})=\hat{\mathbf{j}}_{u}(x)-\phi(x) \hat{\mathbf{j}}(x)$ is convenient for calculations, because the external perturbation appears only in $\rho_{\phi}$, which simplifies the perturbation theory for $\mathbf{J}_{E}=\operatorname{Tr}\left\{\rho_{\phi} \hat{\mathbf{j}}_{E}(\mathbf{x})\right\}$. The statistical averaging of the last term in Eq. (124) gives rise to the Joule heat, which ensures the proper temperature distribution in a stationary flow [3]. We cannot neglect this term, because no matter how small $\nabla \phi(x)$, the value of $\phi(x)$ is arbitrary large for $x \rightarrow \pm \infty$.

\section{Examples of current operators}

As a case study we take the Falicov-Kimball model, Hubbard model and periodic Anderson model with on-site hybridization, $V_{i j}=V \delta_{i j}$. The Hamiltonians for the FalicovKimball and Hubbard models can be summarized by the following form:

$$
\mathscr{H}=-\sum_{i j} t_{i j} c_{i}^{\dagger} c_{j}-\sum_{i j} t_{i j}^{f} f_{i}^{\dagger} f_{j}+U \sum_{i} c_{i}^{\dagger} c_{i} f_{i}^{\dagger} f_{i}
$$

In Equation (125), we have fermionic creation (annihilation) operators $c_{i}^{\dagger}\left(c_{i}\right)$ for conduction electrons, and $U$ is the on-site Coulomb repulsion. In the Falicov-Kimball model, we have $t^{f}=0$ and the $f$-electrons are localized on the lattice. In the Hubbard model, we have $t=t^{f}$ and we identify the $c$ electrons as the up-spin electrons and the $f$ electrons as the down-spin electrons. Equation (125) interpolates continuously between the Falicov-Kimball and Hubbard models.

The periodic Anderson model is written in a standard form,

$$
\begin{aligned}
\mathscr{H} & \left.=-\sum_{i j \sigma} t_{i j} c_{i \sigma}^{\dagger} c_{j \sigma}+f_{i \sigma}^{\dagger} f_{i \sigma}\right)+E_{f} \sum_{i} f_{i \sigma}^{\dagger} f_{i \sigma} \\
& +\sum_{i \sigma}\left(V_{i} c_{i \sigma}^{\dagger} f_{i \sigma}+f_{i \sigma}^{\dagger} c_{i \sigma}\right)+U \sum_{i \sigma} f_{i \uparrow}^{\dagger} f_{i \uparrow} f_{i \downarrow}^{\dagger} f_{i \downarrow} .
\end{aligned}
$$

The conduction and localized electrons as the same as in the Falicov-Kimball model, except now they have spin ( $E_{f}$ is the localized electron site energy). The parameter $V$ is 
the on-site hybridization between conduction and localized electrons on the same site, and $U$ is the on-site Coulomb repulsion between $f$-electrons with different spin.

The Hamiltonians become quite simple in momentum space:

$$
\mathscr{H}=\sum_{\mathbf{k}} \varepsilon(\mathbf{k}) c_{\mathbf{k}}^{\dagger} c_{\mathbf{k}}+\sum_{\mathbf{k}} \varepsilon^{f}(\mathbf{k})+U \sum_{\mathbf{p}, \mathbf{k}, \mathbf{q}} c_{\mathbf{k}-\mathbf{q}}^{\dagger} c_{\mathbf{k}} f_{\mathbf{p}+\mathbf{q}}^{\dagger} f_{\mathbf{p}}
$$

for the Falicov-Kimball-Hubbard model, where the fermionic creation and annihilation operators now create or annihilate electrons with well-defined momentum. The periodic Anderson model becomes

$$
\begin{aligned}
\mathscr{H} & =\sum_{\mathbf{k} \sigma} \varepsilon(\mathbf{k}) c_{\mathbf{k} \sigma}^{\dagger} c_{\mathbf{k} \sigma}+E_{f} \sum_{\mathbf{k} \sigma} f_{\mathbf{k} \sigma}^{\dagger} f_{\mathbf{k} \sigma} \\
& +V \sum_{\mathbf{k} \sigma}\left(c_{\mathbf{k} \sigma}^{\dagger} f_{\mathbf{k} \sigma}+f_{\mathbf{k} \sigma}^{\dagger} c_{\mathbf{k} \sigma}\right)+U \sum_{\mathbf{p}, \mathbf{k}, \mathbf{q}} f_{\mathbf{p}+\mathbf{q} \uparrow}^{\dagger} f_{\mathbf{p} \uparrow} f_{\mathbf{k}-\mathbf{q} \downarrow}^{\dagger} f_{\mathbf{k} \downarrow} .
\end{aligned}
$$

To find the $q=0$ component of the charge-current operator we define the polarization operator $\mathbf{P}_{c}=e \sum_{i} \mathbf{R}_{i} c_{i}^{\dagger} c_{i}$, with the obvious generalization if we need to include spin, and and evaluate the commutator $\left[\mathscr{H}, \mathbf{P}_{c}\right]$. Since the potential energy depends only on the number operators the charge polarization operator commutes with the $U$-term and we need evaluate only the commutator with the kinetic-energy piece of the Hamiltonian, which is straightforward in real space. Converting to a momentum-space representation gives

$$
\mathbf{j}=e \sum_{\mathbf{k}} \mathbf{v}(\mathbf{k}) c_{\mathbf{k}}^{\dagger} c_{\mathbf{k}}
$$

where $\mathbf{v}(\mathbf{k})=\nabla \varepsilon(\mathbf{k})$ is the unrenormalized velocity.

The non-local hybridization would generate the additional term,

$$
\mathbf{j}=e \sum_{\mathbf{k}} \mathbf{v}(\mathbf{k}) c_{\mathbf{k}}^{\dagger} c_{\mathbf{k}}+e \sum_{\mathbf{p q}}\left[\nabla_{\mathbf{p}} V_{\mathbf{p}} c_{\mathbf{p}+\mathbf{q}}^{\dagger} f_{\mathbf{q}}+\nabla_{\mathbf{p}} V_{\mathbf{p}}^{*} f_{\mathbf{q}}^{\dagger} c_{\mathbf{p}+\mathbf{q}}\right]
$$

The energy-current operator is more complicated to construct. The energypolarization operator is $\mathbf{P}_{E}=\sum_{i} \mathbf{R}_{i} h_{i}$, and its proper definition requires assigning an energy operator $h_{i}$ to each lattice site $i\left(\mathscr{H}=\sum_{i} h_{i}\right)$. For a local potential energy, such an assignment is completely straightforward, but the hopping terms in the kinetic energy are more complicated - the standard procedure is to associate one half of the operator that connects sites $i$ and $j$ to the local energy operator at site $i$ and at site $j$. Once $h_{i}$ has been defined, we can calculate the commutator $\left[\mathscr{H}, \mathbf{P}_{E}\right]$ and find the energy-current operator. Unlike the charge current operator, which was essentially independent of the model considered, the energy current operator is different for each Hamiltonian considered. So we summarize the results for each case considered here. For the Falicov-Kimball and Hubbard models, the energy-current operator becomes

$$
\mathbf{j}_{E}^{\mathrm{FK}}=\sum_{\mathbf{k}} \varepsilon(\mathbf{k}) \mathbf{v}(\mathbf{k}) c_{\mathbf{k}}^{\dagger} c_{\mathbf{k}}+\frac{U}{2} \sum_{\mathbf{k} \mathbf{q} \mathbf{q}^{\prime}}\left[\mathbf{v}(\mathbf{q})+\mathbf{v}\left(\mathbf{q}^{\prime}\right)\right] f_{\mathbf{k}}^{\dagger} f_{\mathbf{k}+\mathbf{q}-\mathbf{q}^{\prime}} c_{\mathbf{q}}^{\dagger} c_{\mathbf{q}^{\prime}}
$$


For the Hubbard model it is

$$
\mathbf{j}_{Q}^{\mathrm{H}}=\sum_{\mathbf{k} \sigma} \varepsilon(\mathbf{k}) \mathbf{v}(\mathbf{k}) c_{\mathbf{k} \sigma}^{\dagger} c_{\mathbf{k} \sigma}+\frac{U}{2} \sum_{\mathbf{k q q} \mathbf{q}^{\prime}}\left[\mathbf{v}(\mathbf{q})+\mathbf{v}\left(\mathbf{q}^{\prime}\right)\right] c_{\mathbf{q} \uparrow}^{\dagger} c_{\mathbf{q}^{\prime} \uparrow} c_{\mathbf{k} \downarrow}^{\dagger} c_{\mathbf{k}+\mathbf{q}-\mathbf{q}^{\prime} \downarrow} .
$$

For the periodic Anderson model we have to evaluate (in obvious notation)

$$
\mathbf{j}_{E}=i \sum_{i j} \mathbf{R}_{j}\left[\mathscr{H}_{i}^{T}+\mathscr{H}_{i}^{V}+\mathscr{H}_{i}^{U}, \mathscr{H}_{j}^{T}+\mathscr{H}_{j}^{V}+\mathscr{H}_{j}^{U}\right]=\mathbf{j}_{E}^{T T}+\left(\mathbf{j}_{E}^{T V}+\mathbf{j}_{E}^{V T}\right)
$$

where the last equation holds because $\left[\mathscr{H}_{i}^{T}, \mathscr{H}_{j}^{U}\right]=0$ and

$$
\mathbf{j}_{E}^{V U}+\mathbf{j}_{E}^{U V}=i \sum_{i} \mathbf{R}_{i}\left\{\left[\mathscr{H}_{i}^{V}, \mathscr{H}_{i}^{U}\right]+\left[\mathscr{H}_{i}^{U}, \mathscr{H}_{i}^{V}\right]\right\}=0 .
$$

The kinetic term

$$
\mathbf{j}_{E}^{T T}=i \sum_{i} \mathbf{R}_{i}\left[\mathscr{H}_{i}^{T}, \mathscr{H}_{i}^{T}\right]
$$

is the same as for the Falicov-Kimbal model and we only have to calculate

$$
\mathbf{j}_{E}^{T V}+\mathbf{j}_{E}^{V T}=i \sum_{i} \mathbf{R}_{i}\left[\mathscr{H}_{i}^{T}, \mathscr{H}_{i}^{V}\right]+i \sum_{i} \mathbf{R}_{i}\left[\mathscr{H}_{i}^{V}, \mathscr{H}_{i}^{T}\right]
$$

Direct evaluation in the site representation gives

$$
\mathbf{j}_{E}^{T V}+\mathbf{j}_{E}^{V T}=-i \frac{W}{2} \sum_{i \delta \sigma}\left\{V_{i+\delta}\left(\mathbf{R}_{i+\delta}-\mathbf{R}_{i}\right) c_{i \sigma}^{\dagger} f_{i+\delta \sigma}+V_{i+\delta}^{*}\left(\mathbf{R}_{i+\delta}-\mathbf{R}_{i}\right) c_{i \sigma} f_{i+\delta \sigma}^{\dagger} \cdot\right\}
$$

In the momentum representation this term can be written as

$$
\mathbf{j}_{E}^{T V}+\mathbf{j}_{E}^{V T}=-\frac{1}{2} \sum_{\mathbf{p q} \sigma} \mathbf{v}_{\mathbf{p}}\left\{V_{\mathbf{q}-\mathbf{p}} c_{\mathbf{p} \sigma}^{\dagger} f_{\mathbf{q} \sigma}+V_{\mathbf{p}-\mathbf{q}}^{*} f_{\mathbf{q} \sigma}^{\dagger} c_{\mathbf{p} \sigma}\right\}
$$

For the constant hybridization the $q=0$ Fourier component of the energy density operator is

$$
\mathbf{j}_{E}=\sum_{\mathbf{k}} \varepsilon(\mathbf{p}) \mathbf{v}(\mathbf{p}) c_{\mathbf{p}}^{\dagger} c_{\mathbf{p}}-\frac{V}{2} \sum_{\mathbf{p} \sigma} \mathbf{v}_{\mathbf{p}}\left\{c_{\mathbf{p} \sigma}^{\dagger} f_{\mathbf{p} \sigma}+f_{\mathbf{p} \sigma}^{\dagger} c_{\mathbf{p} \sigma}\right\}
$$

This energy current operator, together with the charge current operator defined by Eq. (128) enters the correlation functions for thermal transport.

\section{MAHAN-JONSON THEOREM}

The Jonson-Mahan[7, 8, 9] theorem provides a simple relation between the transport coefficient for the electrical conductivity and that needed for the thermopower and thermal conductivity. The relation is that the integral for the $L_{12}$ coefficient has an extra power of frequency in the integrand than the $L_{11}$ coefficient and that $L_{22}$ has 
one more power of frequency than $L_{21}$. This result has been known for many years for a noninteracting system - the Jonson-Mahan theorem generalizes this result for a wide class of many-body systems (including the Falicov-Kimball model, the Hubbard model, and the periodic Anderson model. We provide the proof for infinite dimensional lattices[?].

We begin with the generalized two-particle correlation function

$$
F_{\alpha \beta}\left(\tau, \tau^{\prime}, \tau^{\prime \prime}, \tau^{\prime \prime \prime}\right)=\sum_{q q^{\prime} \sigma \sigma^{\prime}} \mathbf{v}_{q \alpha} \mathbf{v}_{q^{\prime} \beta}\left\langle T_{\tau} c_{q \sigma}^{\dagger}(\tau) c_{q \sigma}\left(\tau^{\prime}\right) c_{q^{\prime} \sigma^{\prime}}^{\dagger}\left(\tau^{\prime \prime}\right) c_{q^{\prime} \sigma^{\prime}}\left(\tau^{\prime \prime \prime}\right)\right\rangle,
$$

where $c_{q \sigma}^{\dagger}$ and $c_{q \sigma}$ denote the creation and the annihilation operators for conduction electrons and the averaging is with respect to the interacting Hamiltonian. This function provides all the relevant "polarization operators", which can be shown by a method based on the Heisenberg equation of motion. Using

$$
i \frac{d}{d t} \mathscr{O}_{\mathrm{H}}(t)=\left[\mathscr{O}_{\mathrm{H}}(t), \mathscr{H}_{\mathrm{H}}(t)\right]
$$

we find the time-dependence of the fermionic creation and annihilation operators (in the momentum basis and on the imaginary time-axis). The commutators are straightforward to calculate and become

$$
\left[c_{\mathbf{k}}^{\dagger}(\tau), \mathscr{H}\right]=-\varepsilon(\mathbf{k}) c_{\mathbf{k}}^{\dagger}(\tau)-U \sum_{\mathbf{p}, \mathbf{q}} f_{\mathbf{p}+\mathbf{q}}^{\dagger}(\tau) f_{\mathbf{p}}(t) c_{\mathbf{k}-\mathbf{q}}^{\dagger}(\tau)
$$

for the Falicov-Kimball-Hubbard model and

$$
\left[c_{\mathbf{k} \sigma}^{\dagger}(\tau), \mathscr{H}\right]=-\varepsilon(\mathbf{k}) c_{\mathbf{k} \sigma}^{\dagger}(\tau)-\sum_{\mathbf{p}} V_{\mathbf{p}}^{*} f_{\mathbf{k}-\mathbf{p} \sigma}^{\dagger}(\tau)
$$

for the periodic Anderson model. Note, $\left[c_{\mathbf{k}}^{\dagger}, H_{U}\right]=0$ for PAM. The commutator for the $c$ operators is similar to evaluate. This shows that

$$
\lim _{\tau^{\prime} \rightarrow \tau^{-}} \frac{1}{2}\left(\frac{\partial}{\partial \tau}-\frac{\partial}{\partial \tau^{\prime}}\right) \sum_{q \sigma} \mathbf{v}_{q} c_{q \sigma}^{\dagger}(\tau) c_{q \sigma}\left(\tau^{\prime}\right)=\mathbf{j}_{Q}(\tau) .
$$

The Jonson-Mahan theorem will hold for any Hamiltonian that satisfies Eq. (138). In particular, it holds true for the Falicov-Kimball-Hubbard model and the periodic Anderson model[9] and allows us to express the "polarization operators" in terms of the generalized function $F$ and its time-derivatives. We obtain

$$
\mathscr{L}_{11}=\pi T e^{2} \int_{0}^{\beta} d \tau e^{i v_{l} \tau} F\left(\tau, \tau^{-}, 0,0\right),
$$

for the conductivity,

$$
\mathscr{L}_{12}=\pi T e \int_{0}^{\beta} d \tau e^{i v_{l} \tau} \frac{1}{2}\left(\frac{\partial}{\partial \tau}-\frac{\partial}{\partial \tau^{\prime}}\right) F\left(\tau, \tau^{\prime}, 0,0\right),
$$


(in the limit where $\tau^{\prime} \rightarrow \tau^{-}$) for the thermopower, and

$$
\mathscr{L}_{22}=\pi T \int_{0}^{\beta} d \tau e^{i v_{l} \tau} \frac{1}{4}\left(\frac{\partial}{\partial \tau}-\frac{\partial}{\partial \tau^{\prime}}\right)\left(\frac{\partial}{\partial \tau^{\prime \prime}}-\frac{\partial}{\partial \tau^{\prime \prime \prime}}\right) \times F\left(\tau, \tau^{\prime}, \tau^{\prime \prime}, \tau^{\prime \prime \prime}\right)
$$

(in the limit where $\tau^{\prime} \rightarrow \tau^{-}, \tau^{\prime \prime \prime} \rightarrow \tau^{\prime \prime}$, and $\tau^{\prime \prime} \rightarrow 0^{+}$) for the thermal conductivity.

The analytic continuation to the real axis is quite complicated in general[? ] and we restrict our considerations to the infinite-dimensional limit. In that case the two-particle correlation function is expressed by just its bare bubble because the irreducible charge vertex has a different symmetry than $\mathbf{v}_{q}$. Hence, we immediately learn that

$$
F_{\alpha \beta}\left(\tau, \tau^{\prime}, \tau^{\prime \prime}, \tau^{\prime \prime \prime}\right)=-\sum_{q \sigma} \mathbf{v}_{q \alpha}^{2} \delta_{\alpha \beta} G_{q \sigma}\left(\tau^{\prime \prime \prime}-\tau\right) G_{q \sigma}\left(\tau^{\prime}-\tau^{\prime \prime}\right)
$$

where $G_{q \sigma}(\tau)=-\left\langle T_{\tau} c_{q \sigma}(\tau) c_{q \sigma}^{\dagger}(0)\right\rangle$ is the imaginary time Green's function of conduction electrons. Next, we need to determine a spectral representation for the Green's function. Using the fact that

$$
G_{q \sigma}(z)=-\frac{1}{\pi} \int d \omega \frac{\operatorname{Im} G_{q \sigma}(\omega)}{z-\omega}
$$

with $z$ in the upper half plane (which can be shown by using the Lehmann representation), we find that

$$
G_{q \sigma}(\tau)=-\frac{1}{\pi} \int d \omega T \sum_{n} \frac{e^{-i \omega_{n} \tau}}{i \omega_{n}-\omega} \operatorname{Im} G_{q \sigma}(\omega)
$$

Now we convert the sum over Matsubara frequencies into a contour integral (that surrounds each Matsubara frequency, but does not cross the real axis-the contour is then deformed into two contours, one running just above and the other just below the real axis), but we must be careful to ensure that the procedure is well-defined. If $\tau<0$, then

$$
\begin{aligned}
T \sum_{n} \frac{e^{-i \omega_{n} \tau}}{i \omega_{n}-\omega} & =-\frac{i}{2 \pi} \int_{C} d z \frac{e^{-z \tau}}{z-\omega} f(z) \\
& =-\frac{i}{2 \pi} \int_{-\infty}^{\infty} d z e^{-z \tau} f(z)\left[\frac{1}{z+i 0^{+}-\omega}-\frac{1}{z-i 0^{+}-\omega}\right] \\
& =-e^{-\omega \tau} f(\omega)
\end{aligned}
$$

This result is well-defined because the Fermi factor provides convergence (asymptotically like $\exp [-\beta z]$ ) for $z \rightarrow \infty$ and the $\exp [-z \tau]$ term provides boundedness for $z \rightarrow-\infty$ when $\tau<0$. Since $1-f(z)$ has the same poles as $f(z)$ on the imaginary axis, with residues that have the opposite sign, and it behaves like $\exp [\beta z]$ for $z \rightarrow-\infty$, one finds

$$
T \sum_{n} \frac{e^{-i \omega_{n} \tau}}{i \omega_{n}-\omega}=e^{-\omega \tau}[1-f(\omega)]
$$


for $\tau>0$. The results in Eqs. (145) and (146) can then be substituted into Eq. (144) to get the final formula for the Green's function

$$
G_{q \sigma}(\tau)= \begin{cases}\int d \omega A_{q \sigma}(\omega) e^{-\omega \tau}[1-f(\omega)], & \tau>0 \\ \int d \omega A_{q \sigma}(\omega) e^{-\omega \tau}[-f(\omega)], & \tau<0\end{cases}
$$

where $A_{q \sigma}(\omega)=-\operatorname{Im} G_{q \sigma}(\omega) / \pi$ is the spectral function. Substituting into Eq. (142), then yields

$$
\begin{aligned}
F_{\alpha \beta}\left(\tau, \tau^{\prime}, \tau^{\prime \prime}, \tau^{\prime \prime \prime}\right) & =\delta_{\alpha \beta} \int d \varepsilon \mathbf{v}_{\varepsilon}^{2} \rho(\varepsilon) \int d \omega \int d \omega^{\prime} \\
& \times A(\varepsilon, \omega) A\left(\varepsilon, \omega^{\prime}\right) e^{\omega\left(\tau-\tau^{\prime \prime \prime}\right)-\omega^{\prime}\left(\tau^{\prime}-\tau^{\prime \prime}\right)} f(\omega)\left[1-f\left(\omega^{\prime}\right)\right]
\end{aligned}
$$

where we replaced the $q$-summation by an integral over the non-interacting density of states. Because of Eq. (148), the analytic continuation of the correlation function given by Eq. (139) is trivial (one first converts from imaginary time to Matsubara frequencies and then performs the Wick rotation to the real frequency axis), and if we note the identity

$$
f(\omega)-f(\omega+v)=-f(\omega)[1-f(\omega+v)]\left[e^{-\beta v}-1\right],
$$

then we can easily compute that

$$
\bar{L}_{11}=T e^{2}\left\langle\mathbf{v}_{\mathbf{k}}^{2}\right\rangle_{\mathrm{F} S} \int d \omega \int d \varepsilon \rho(\varepsilon)\left(-\frac{d f(\omega)}{d \omega}\right) A^{2}(\varepsilon, \omega),
$$

for the conductivity,

$$
\bar{L}_{12}=T e\left\langle\mathbf{v}_{\mathbf{k}}^{2}\right\rangle_{\mathrm{FS}} \int d \omega \int d \varepsilon \rho(\varepsilon)\left(-\frac{d f(\omega)}{d \omega}\right) A^{2}(\varepsilon, \omega) \omega,
$$

for the thermopower, and

$$
\bar{L}_{22}=T\left\langle\mathbf{v}_{\mathbf{k}}^{2}\right\rangle_{\mathrm{FS}} \int d \omega \int d \varepsilon \rho(\varepsilon)\left(-\frac{d f(\omega)}{d \omega}\right) A^{2}(\varepsilon, \omega) \omega^{2},
$$

for the thermal conductivity. Here, $\left\langle\mathbf{v}_{\mathbf{k}}^{2}\right\rangle_{\mathrm{FS}}$ denotes the square of conduction electron's velocity averaged over the renormalized Fermi surface (FS). This pre-factor has been taken out of the integral because $(-d f(\omega) / d \omega)$ restricts the integration region in the $q$-space to the vicinity of the Fermi surface. This proves Mott's form for the thermal transport.

For the degenerate models with $N$ conduction channels it is convenient to rewrite the above expressions in terms of the Luttinger's coefficients $L_{12}=\bar{L}_{12} / T$ defined by Eq. (21). This gives $\sigma(T)=e^{2} N L_{11}$ for the charge conductivity, $\alpha(T)|e| T=-L_{12} / L_{11}$ for the thermopower, and $\kappa(T) T=N\left(L_{22}-L_{12}^{2} / L_{11}\right)$ for the electronic contribution to thermal conductivity, where

$$
L_{m n}=\left\langle\mathbf{v}_{\mathbf{k}}^{2}\right\rangle_{\mathrm{FS}} \int d \omega\left(-\frac{d f}{d \omega}\right) \omega^{m+n-2} \Lambda(\omega, T) .
$$


$f(\omega)=1 /[1+\exp (\beta \omega)]$ is the Fermi-Dirac distribution function, $\omega$ is measured with respect to the chemical potential $\mu$, and $\Lambda(\omega, T)$ is the frequency-dependent correlation function

$$
\Lambda(\omega, T)=\int d \varepsilon \rho(\varepsilon) A^{2}(\varepsilon, \omega)
$$

calculated for a single channel. For the infinite-dimensional Falicov-Kimball-Hubbard model and the Anderson model with the Gaussian non-interacting density of states the transport relaxation time can be given an explicit form. Using the Dyson equation for the conduction electrons Green's function

$$
G_{q \sigma}(\omega)=\frac{1}{\omega+\mu-\Sigma(\omega)-\varepsilon(q)}
$$

and the fact that the self energy is momentum independent we can evaluate the integral in Eq. (154) with the result

$$
\Lambda(\omega)=\frac{\operatorname{Im} G(\omega)}{\operatorname{Im} \Sigma(\omega)}+2-2 \operatorname{Re}\{[\omega+\mu-\Sigma(\omega)] G(\omega)\}
$$

This expression simplifies at low temperatures, where the frequency integration in Eq. (153) is restricted by $(-d f / d \omega)$ to the Fermi window, $|\omega| \leq k_{B} T$. In the $T \rightarrow 0$ limit we can write Eq. (154) as

$$
\Lambda(\omega, T)=\mathscr{N}_{c}(\omega) \tau(\omega, T)
$$

where $\mathscr{N}_{c}(\omega)=\sum_{\mathbf{k}} A_{\mathbf{k}}(\omega)$ is the renormalized DOS of conduction electrons, and $\tau(\omega, T)$ the transport relaxation time

$$
\tau(\omega, T)=\frac{-1}{\operatorname{Im} \Sigma_{c}\left(\omega^{+}, T\right)}
$$

which is given by the momentum-independent self energy of $c$ electrons. In deriving Eq. (156) we included only the divergent term in $\Lambda(\omega, T)$, and dropped the finite term which contributes to the full DMFT expression but does not affect the $T, \omega \rightarrow 0$ results. To calculate the renormalized expressions for $v_{k_{F}}^{2}, \mathscr{N}_{c}(\omega), \tau(\omega)$, we make the approximations which are consistent with this derivation.

\section{MICROSCOPIC SOLUTION FOR TRANSPORT COEFFICIENTS}

We now discus the thermoelectric properties of intermetallic compounds with Cerium, Europium or Ytterbium ions using the theoretical methods explained in the previous sections. We summarize, first, the transport properties of heavy fermions and valence fluctuators and, then, describe the anomalies observed in $\mathrm{YbInCu}_{4}$-like systems which show a valence-change transition. The thermal transport of heavy fermions and valence fluctuators is explained by the periodic Anderson model and that of the $\mathrm{YbInCu}_{4}$-like systems by the Falicov-Kimball model. 


\section{Experimental summary}

The experimental data on heavy fermions and valence fluctuators can be summarized as follows. The thermoelectric power $\alpha(T)$ exhibits the characteristic features that allow the classification of these compounds into several distinct groups[10, 11, 12]. In the case of Cerium ions, the compounds belonging to the first group (type (a) systems) have the thermopower with a deep negative minimum at low temperatures $[10,11,13$, $14,15]$ and a high-temperature maximum, typically between $100 \mathrm{~K}$ and $300 \mathrm{~K}$. At the maximum, $\alpha(T)$ could be either positive or negative. At very low temperatures, the type (a) systems order magnetically or become superconducting. The compounds of the second group (type (b) systems) have a negative low-temperature minimum and a positive high-temperature maximum but, in addition, the thermopower shows a smaller positive peak at lowest temperatures.[16, 17, 10, 18, 19] This second peak is sometimes concealed by a low-temperature phase transition; for example, in $\mathrm{CeCu}_{2} \mathrm{Si}_{2}$ it becomes visible only in an external magnetic field which suppresses the superconducting transition,[13] and in $\mathrm{CeRu}_{2} \mathrm{Ge}_{2}$ it shows up when the external pressure suppresses the magnetic transition.[20] The experimental evidence is now accumulating that the initial slope of the thermopower $\alpha(T) / T$ is positive for this class of (heavy fermion) materials, provided the measurements are performed at low enough temperature and with sufficient accuracy.[18, 19, 15, 21] In the third group (type (c) systems), the lowtemperature peak is well pronounced and shifted towards the high-temperature peak. The main difference with respect to the type (b) systems is that the sign-change of $\alpha(T)$ does not occur.[22, 23, 24, 25] Finally, in some cases (type (d) systems) the thermopower grows monotonically towards the high-temperature maximum, and the low-temperature structure appears only as a shoulder on a broad peak, or is not resolved at all. $[26,23,27,19]$

The clue to these various types of behavior comes from the high-pressure[28, 29, $20,30,11]$ and doping studies, [10, 23, 24, 31, 32, 33, 34, 35] which show that the thermopower of Cerium compounds changes continuously from type (a) to type (d). A typical example is provided by the shape of $\alpha(T)$ observed in $\mathrm{CeRu}_{2} \mathrm{Ge}_{2}$ at various pressures.[20] At ambient pressure, $\mathrm{CeRu}_{2} \mathrm{Ge}_{2}$ is a type (a) system with a magnetic ground state and negative thermopower below $300 \mathrm{~K}$. An increase of pressure leads to a thermopower with a small positive peak at low temperatures and an enhanced peak at high temperatures. A further increase of pressure enhances both peaks, shifts the low-temperature peak towards the high-temperature one, and makes the thermopower at intermediate temperatures less negative. For large enough pressure, the sign-change does not occur at all and for very high pressure the low-temperature peak merges with the high-temperature one, and transforms into a shoulder. The high-temperature peak grows continuously but its position remains more or less constant, as $\alpha(T)$ changes from type (a) to (c). Eventually, for pressures above $10 \mathrm{GPa}$, the $\alpha(T)$ assumes the (d) shape. Here, the initial slope of $\alpha(T)$ decreases and the position of the maximum shifts to higher temperatures, but its magnitude does not change as pressure increases. Similar behavior is also seen in the high-pressure data of, $\mathrm{CeCu}_{2} \mathrm{Si}_{2}$, [28] $\mathrm{CeCu}_{2} \mathrm{Ge}_{2}$, [30, 36] or $\mathrm{CePd}_{2} \mathrm{Si}_{2}$.[37] As regards doping, the chemical substitutions which reduce the volume and make Ce ions less magnetic, transform $\alpha(T)$ from type (a) to type (b),[31] from (b) to (c), $[23,24]$ or from (a) to(c), $[32,33,34]$ while the substitutions which expand 
the volume and make the Ce more magnetic, transform the thermopower from, say, type (d) to type (c) or from type (c) to type (b).[35] This variation of shape is an indication that the local environment plays an important role in determining the magnetic character of $\mathrm{Ce}$ and $\mathrm{Yb}$ ions. Even at high temperatures, where each $4 f$ ion is an independent scatterer, the thermopower of a sample with a high concentration of $4 f$ ions cannot be obtained by rescaling the low-concentration data.

The Ytterbium intermetallics can be classified using the mirror-image analogy with Cerium systems. This holds because the $\mathrm{Yb}$ ions fluctuate between $4 f^{13}$ and $4 f^{14}$, while the Ce ions fluctuate between $4 f^{1}$ and $4 f^{0}$ configurations, and the dynamics of a single $f$ hole and a single $f$ electron is the same. A well-defined local moment leads in $\mathrm{Yb}$ systems to the type (a) behavior, such that the thermopower has a negative minimum at high temperatures and a positive maximum at low temperatures; $[38,39]$ the size of the minimum is about the same as the size of the maximum. The thermopower of (b)-type Yb systems[40, 41, 42] mirrors the (b)-type Ce systems. Here, one finds two negative minima separated by a small positive maximum. The type (c) Yb systems have a nonmonotonic thermopower with a large (negative) minimum at high temperatures and a smaller one at low temperatures, but there is no sign-change.[40, 42, 38, 41] Finally, the thermopower with a single negative peak centered around $100 \mathrm{~K}$ [43, 40, 27, 38] mirrors the type (d) behavior of Ce systems. The reduction of volume by pressure or doping $[42,38]$ stabilizes the magnetic $4 f^{13}$ configuration of $\mathrm{Yb}$ ions, and transforms $\alpha(T)$ from, say, type (b) to type (a), from (c) to (b), or from (c) to (a).

The experimental results show that $4 f$ systems with similar thermopowers exhibit similarities in other thermodynamic [44] and transport[45, 46, 47] properties, and there is an obvious correlation between the shape of $\alpha(T)$ and the magnetic character of the $4 f$ ions. The thermopower measurements [48] provide a simple and sensitive tool for characterizing the magnetic state of a $4 f$ ion in a given metallic matrix: the shape of $\alpha(T)$ changes from the (a)-type in the case of magnetic Ce $(\mathrm{Yb})$ ions with stable $\mathrm{f}^{1}\left(4 f^{13}\right)$ configuration to the (d)-type for non-magnetic $\mathrm{Ce}(\mathrm{Yb})$ ions which fluctuate between the $4 f^{1}\left(4 f^{13}\right)$ and $4 f^{0}\left(4 f^{14}\right)$ configurations.

At very low temperatures one finds additional interestig features. The data show [21, 49] a striking correlation between the low-temperature Seebeck coefficient $\alpha$ and the specific heat coefficient $\gamma=C_{V} / T$; the ratio $q=|e| \lim _{T \rightarrow 0} \alpha / \gamma T$ is nearly constant, although the absolute values of $\gamma$ and $\alpha / T$ vary by orders of magnitude. The $q$ ratio increases as the number of conduction electrons $n_{c}$ decreases and becomes large in bad metals[21, 49]. The Kadowaki-Woods (KW) ratio [50] which is defined as $\rho(T) /(\gamma T)^{2}$, where $\rho(T)$ is the electrical resistivity, exhibits similar universal features, if one takes into account the effective low-temperature degeneracy of the $f$ states $[51,52]$. The near constancy of the KW and $q$ ratios suggests a 'universal law' for the low-temperature power factor $P=\alpha^{2} / \rho$ and brings to the fore the validity of the Wiedemann-Franz (WF) law, $\kappa \rho / T=\mathscr{L}_{0}$, and a possibility of enhancing the electronic thermoelectric figure-of-merit in strongly correlated materials, $Z T=\alpha^{2} T / \kappa \rho$, where $\kappa$ is the electronic contribution to the thermal conductivity and $\mathscr{L}_{0}=\pi^{2} k_{B}^{2} / 3 e^{2}$ the Fermi liquid (FL) Lorenz number. When the WF law holds, metals must have a thermopower larger than $155 \mu \mathrm{V} / \mathrm{K}$ to achieve $Z T>1$; to date no metal has been found with so large a thermopower. In the temperature window where the effective Lorenz number $(\mathscr{L}=$ $\kappa \rho / T$ ) is reduced, one can achieve $Z T>1$ with substantially lower thermopowers, 
which might make it possible to find strongly correlated metals that can be used for cooling applications at low temperature.

Finally, we mention a new class of materials in which the transition between the high-entropy phase and the low-entropy does no follow the usual "Kondo route" but is driven by the valence-change transition, as observed in $\mathrm{EuCu}_{2} \mathrm{Ni}_{2}, \mathrm{YbIn}_{1-x} \mathrm{Ag}_{x} \mathrm{Cu}_{4}$ or $\mathrm{YbInCu}_{4}[53,54]$. The main features of these systems are best revealed by taking $\mathrm{YbIn}_{1-x} \mathrm{Ag}_{x} \mathrm{Cu}_{4}$ as an example. Indium doping expands the lattice and increases the weight of $\mathrm{Yb}^{2+}$ with respect to $\mathrm{Yb}^{3+}$ configuration[55] by transferring the electrons from the conduction band to the $4 f$-state. Thus, chemical pressure reduces the number of $f$-holes and increases the Kondo coupling which makes the compound less magnetic and increases charge fluctuations. For $x \geq 0.5$, the compound is a typical heavy fermion in which the substitution of the monovalent Ag by the trivalent In compensates the chemical pressure effects, such that the total number of conduction electrons increases. For for $x \leq 0.5$, the Ag-In substitution brings eventually $\mu$ and $E_{f}$ in the vicinity of the band edge $E_{c}$ which gives rise to completely new features. In this concentration range $\mathrm{YbIn}_{1-x} \mathrm{Ag}_{x} \mathrm{Cu}_{4}$ is already a valence fluctuator with temperature dependent $n_{f}^{h}(T)$, such that the temperature-induced transfer of $f$-holes in the conduction band can reduce $\mu-E_{c}$ and $E_{f}-E_{c}$ to zero. Once $\mu$ is within the gap of the density of states, the effective hybridization is switched off and the magnetic moment of the $f$-ions cannot be quenched by Kondo screening. Thus, the transition from the low-temperature coherent FL state to the high-temperature disordered paramagnetic state cannot follow the usual 'Kondo route', taken by the heavy fermions. The valence fluctuators like $\mathrm{YbIn}_{1-x} \mathrm{Ag}_{x} \mathrm{Cu}_{4}$ for $x \leq 0.5$, belong to a new class of materials in which the transition between the lowand high-entropy phase is driven by the Falicov-Kimball interaction. This gives rise, at the temperature $T_{V}$, to a change in the relative occupancy of the $f$ and the conduction states and an abrupt modification of the properties of the system. The valence change transition is clearly seen in the XPS data; above $T_{V}$, the spectra indicate a stable $4 f^{13}$ configuration of $\mathrm{Yb}$ ions and below $T_{V}$ one has a mixture of $4 f^{13}$ and $4 f^{14}$ states. The magnetic character of the $\mathrm{Yb}$ ions changes at $T_{V}$, as indicated by an abrupt change of the susceptibility from Pauli-like to Curie-like. In the high-temperature phase, the Curie constant is close to the free ion value of $\mathrm{Yb}^{3+}$. The conduction states are also modified at $T_{V}$, as indicated by a drastic change of the frequency dependence of the optical conductivity and by a large increase in the resistivity[55]. The electrical resistance and the Hall coefficient of the high-temperature phase of $\mathrm{YbIn}_{1-x} \mathrm{Ag}_{x} \mathrm{Cu}_{4}$ are typical of narrow-band semiconductors, or semi-metals with a very low carrier density, and neither the transport nor the thermodynamic properties show any sign of the Kondo effect. The proximity of $\mu$ to $E_{c}$ is indicated in $\mathrm{YbInCu}_{4}$ by the Hall data and bandstructure calculations[55].

\section{Periodic Anderson model}

We now discuss the thermoelectric properties of heavy fermions and valence fluctuators using the periodic Anderson model. In the first part of this subsection we derive the Fermi liquid laws and explain the universal low-temperature behavior. In the second part 
we discuss the anomalies that one finds in the thermopower of heavy fermions above the Kondo temperature.

\section{Fermi liquid solution}

The coherent thermal transport of heavy fermions and valence fluctuators is explained at low temperatures by the periodic Anderson model with $S U(N)$ symmetry in infinite dimensions. We consider the model with the on-site hybridization, $V_{i j}=V \delta_{i j}$, such that the charge current operators are given by Eqs. (128) and (133). This model satisfies the Jonson-Mahan theorem. Since the integral in Eq. (153) is restricted by $(-d f / d \omega)$ to $|\omega| \leq k_{B} T$, we write in the $T \rightarrow 0$ limit $\Lambda(\omega, T)=\mathscr{N}_{c}(\omega) \tau(\omega, T)$ and calculate the renormalized density of states $\mathscr{N}_{c}(\omega)$ and the transport relaxation time $\tau(\omega, T)$ by the dynamical mean field approximation (DMFT).

The renormalized $c$ and $f$ Green's functions of the periodic Anderson model satisfy the Dyson equations $[56,57]$

$$
G_{c}(\mathbf{k}, z)=\frac{z-\tilde{\varepsilon}_{f}(\omega, T)+\mu}{\left[z-\varepsilon_{\mathbf{k}}+\mu\right]\left[z-\tilde{\varepsilon}_{f}(\omega, T)+\mu\right]-V^{2}},
$$

and

$$
G_{f}(\mathbf{k}, z)=\frac{z-\varepsilon_{\mathbf{k}}+\mu}{z-\tilde{\varepsilon}_{f}(\omega, T)+\mu} G_{c}(\mathbf{k}, z),
$$

where $\tilde{\varepsilon}_{f}(\omega, T)=E_{f}+\operatorname{Re} \Sigma_{f}\left(\omega^{+}, T\right)$ is the renormalized position and $\Sigma_{f}(z)$ the DMFT self-energy $f$ electrons, which is momentum-independent. The retarded (advanced) Green's functions are defined for $z$ in the upper (lower) part of the complex plane. The $c$ electrons self-energy on the real $\omega$-axis is

$$
\Sigma_{c}\left(\omega^{+}\right)=\frac{V^{2}}{\omega^{+}-\tilde{\varepsilon}_{f}(\omega, T)+\mu-\operatorname{Im} \Sigma_{f}\left(\omega^{+}, T\right)},
$$

where $\omega^{ \pm}=\lim _{\delta \rightarrow 0}(\omega \pm i \delta)$.

The singularities of the of $G_{c}(\mathbf{k}, z)$ and $G_{f}(\mathbf{k}, z)$ are defined by the equation

$$
\left(\omega-\varepsilon_{\mathbf{k}}+\mu\right)\left[\omega-\tilde{\varepsilon}_{f}(\omega)+\mu\right]-V^{2}=0,
$$

which gives the QP excitations and provides for $\omega=0$ the renormalized FS of hybridized states. Since $\Sigma_{f}\left(\omega^{+}, T\right)$ is momentum independent in the DMFT approximation, the shape of the FS is determined by the non-interacting dispersion of $c$ states. Using the Luttinger's theorem, which requires that the $k$-space volume enclosed by the FS yields the total concentration of electrons $n=n_{c}+n_{f}$ in each channel, we obtain the FS from the equation $\varepsilon_{\mathbf{k}}=\mu_{0}$, where $\mu_{0}$ is the solution of $n=\int_{-\infty}^{\mu_{0}} d \varepsilon \mathscr{N}_{c}^{0}(\varepsilon)$. Thus, the renormalized FS coincides with the non-interacting one corresponding to $n_{c}+n_{f} c$ electrons and the FS average of $v^{2}$ is given by the integral

$$
v_{k_{F}}^{2}=\int d^{d} \mathbf{k} \delta\left(\varepsilon_{\mathbf{k}}-\mu_{0}\right) v_{\mathbf{k}}^{2} .
$$


The numerical calculations for the 3-dimensional periodic Anderson model with the nearest-neighbor hopping on a simple cubic lattice give $v_{k_{F}}^{2}=\left(t a_{l} / \hbar\right)^{2} v^{2}$ where $v^{2} \simeq 1.4$ for $n \simeq 1 / 2$ and $v^{2} \ll 1$ for $n \simeq 1$.

The renormalized spectral properties around the Fermi level are obtained from the Luttinger theorem. First, following [56], we expand $\operatorname{Re} \Sigma_{f}\left(\omega^{+}, T\right)$ up to the terms linear in $\omega$ which gives $\omega-\left[\tilde{\varepsilon}_{f}(\omega)-\mu\right] \simeq\left(\omega-\tilde{\omega}_{f}\right) Z_{f}^{-1}$. Here, $\tilde{\omega}_{f}$ is the solution of the equation $\tilde{\omega}-\left[\tilde{\varepsilon}_{f}(\tilde{\omega})-\mu\right]=0$ and $Z_{f}^{-1}=\left[1-\partial \Sigma_{f} / \partial \omega\right]_{\omega=0}$ is the enhancement factor. For $\mathbf{k}$ close to the FS and small $\omega$, Eq.(161) gives the QP excitations $\omega=\Omega_{\mathbf{k}}^{ \pm}$, where

$$
\Omega_{\mathbf{k}}^{ \pm}=\frac{1}{2}\left[\left(\varepsilon_{\mathbf{k}}-\mu+\tilde{\omega}_{f}\right) \pm \sqrt{\left(\varepsilon_{\mathbf{k}}-\mu-\tilde{\omega}_{f}\right)^{2}+4 \tilde{V}^{2}}\right],
$$

and $\tilde{V}=V \sqrt{Z_{f}}$ denotes the renormalized hybridization. To describe the excitations in intermetallic compounds with $\mathrm{Ce}(\mathrm{Yb})$ ions we put the chemical potential in the lower (upper) QP branch. The singular parts of the spectral functions can be written as

$$
\begin{aligned}
& A_{c}\left(\varepsilon_{\mathbf{k}}, \omega\right) \simeq a_{\mathbf{k}}^{c}\left(\Omega_{\mathbf{k}}^{ \pm}\right) \delta\left(\omega-\Omega_{\mathbf{k}}^{ \pm}\right), \\
& A_{f}\left(\varepsilon_{\mathbf{k}}, \omega\right) \simeq a_{\mathbf{k}}^{f}\left(\Omega_{\mathbf{k}}^{ \pm}\right) \delta\left(\omega-\Omega_{\mathbf{k}}^{ \pm}\right),
\end{aligned}
$$

where the coefficients are the residues of the QP poles,

$$
a_{\mathbf{k}}^{c}(\omega)=\left[1+Z_{f}^{-1} \frac{V^{2}}{\omega-\tilde{\varepsilon}_{f}(\omega)+\mu}\right]_{\omega=\Omega_{\mathbf{k}}^{ \pm}},
$$

and

$$
a_{\mathbf{k}}^{f}(\omega)=\frac{\left(\omega-\varepsilon_{\mathbf{k}}+\mu\right)^{2}}{V^{2}} a_{\mathbf{k}}^{c}(\omega)
$$

The renormalized $c$ and $f$ DOS are given at low energies by the expressions $[58,59]$

$$
\begin{aligned}
& \mathscr{N}_{c}(\omega) \simeq \mathscr{N}_{c}^{0}\left(\omega+\mu-\frac{V^{2}}{\omega-\tilde{\varepsilon}_{f}(\omega)+\mu}\right), \\
& \mathscr{N}_{f}(\omega) \simeq \frac{\mu-\varepsilon_{\mathbf{k}_{\mathbf{F}}}}{\mu-\tilde{\varepsilon}_{f}(0)} \mathscr{N}_{c}(\omega)
\end{aligned}
$$

where $\mathbf{k}_{\mathbf{F}}$ is Fermi momentum and $\mathscr{N}_{c}^{0}(\omega)$ is the unrenormalized $c$-DOS, taken as a symmetric function of half-width $D$. Using Eq. (161) this can also be written as,

$$
\mathscr{N}_{f}(0)=\frac{V^{2}}{\left[\mu-\tilde{\varepsilon}_{f}(0)\right]^{2}} \mathscr{N}_{c}^{0}\left(\varepsilon_{\mathbf{k}_{\mathbf{F}}}\right)
$$

The total DOS is obtained by multiplying the above expressions by the degeneracy $N$.

The physical interpretation of $\tilde{\omega}_{f}=\left[\tilde{\varepsilon}_{f}(0)-\mu\right] Z_{f}$ follows from the DMFT requirement that the local Green's function of the Anderson lattice coincides with the Green's function of an auxiliary Anderson impurity, such that

$$
\sum_{\mathbf{k}} G_{f}(\mathbf{k}, z)=\frac{1}{z-E_{f}+\mu-\Delta(z)-\Sigma_{f}(z)}=G_{f}^{a u x}(z) .
$$


The auxiliary model describes $f$ electrons distributed over $N$ states and hybridized with an effective $c$ band, The effective hybridization $\Delta(z)$ follows from the condition that the self energy of the lattice and the impurity defines the same functional $\Sigma_{f}\left[G_{f}(z)\right]$. The unrenormalized impurity $f$ level is at $E_{f}$ and in the $\omega, T \rightarrow 0$ limit we approximate $\Delta(\omega) \simeq \Delta(0)=i \Delta_{0}$ with $\Delta_{0}<0$. The DMFT solution requires $\mathscr{N}_{f}(\omega)=A_{f}(\omega)$, where $A_{f}(\omega)=-\mathrm{I} m G_{f}^{\text {aux }}\left(\omega^{+}\right) / \pi$ is the auxiliary spectral function. Keeping only the real part of $\Sigma_{f}(\omega, T)$ we write the low-frequency limit of the renormalized $f$-DOS as

$$
\mathscr{N}_{f}(\omega)=\frac{1}{\pi\left|\Delta_{0}\right|} \frac{\left(\tilde{\Delta}_{f}\right)^{2}}{\left(\omega-\tilde{\omega}_{f}\right)^{2}+\left(\tilde{\Delta}_{f}\right)^{2}},
$$

where $\tilde{\Delta}_{f} \simeq-\Delta_{0} Z_{f}$. The above expression, which holds for $\omega \ll \tilde{\omega}_{f}$, shows that the lattice $f$-DOS can be approximated by the Kondo resonance centered at $\tilde{\omega}_{f}$ and of the width $\tilde{\Delta}_{f}$.

Luttinger's theorem and the DMFT self-consistency condition can now be used to relate $\tilde{\omega}_{f}$ or $\tilde{\Delta}_{f}$ to $\mathscr{N}_{f}(0)$. The QP branches $\Omega_{\mathbf{k}}^{+}$and $\Omega_{\mathbf{k}}^{-}$are separated by the hybridization gap $2 \tilde{V}_{f}$. Close to the Brillouin zone boundary (ZB) and the zone center (ZC), we have $\Omega_{\mathbf{k}}^{+}-\Omega_{\mathbf{k}}^{-} \simeq D\left[1+2(\tilde{V} / D)^{2}\right] \simeq D$. At the FS we have $\Omega_{\mathbf{k}_{F}}^{-}=0$ or $\Omega_{\mathbf{k}_{F}}^{+}=0$, depending on the value of $n_{c}+n_{f}$. Close to the FS, the QP dispersion is very weak and $\Omega_{\mathbf{k}}^{ \pm}$describe two heavy QP bands of half-width $\tilde{V}_{f}^{2} / D$. Taking $n<<2$ for Ce and Eu systems, such that the chemical potential is close to the top of the lower QP branch, we find from $\Omega_{\mathbf{k}_{F}}^{-}=0$ that $\mathbf{k}_{F}$ is close to the ZB, such that $\varepsilon_{\mathbf{k}_{F}}-\mu \simeq D$ and $\mathscr{N}_{c}^{0}\left(\varepsilon_{\mathbf{k}_{F}}\right) D \simeq n_{c} / a \pi N$. The factor $a \pi$ in the denominator, approximately accounts for the fact that the unperturbed $c$-DOS near the edge of the band is smaller than the average unperturbed DOS over the whole energy interval under consideration. For $\mathrm{Yb}$ compounds, we restrict $n_{f}^{h}<<1$ and assume that the system is more than half-filled, such that the lower QP branch is full and the upper branch is fractionally occupied. The equation $\Omega_{\mathbf{k}_{F}}^{+}=0$ gives $\mathbf{k}_{F}$ close to the zone center, such that $\varepsilon_{\mathbf{k}_{F}}-\mu \simeq-D$ and $\mathscr{N}_{c}^{0}\left(\varepsilon_{\mathbf{k}_{F}}\right)\left(\varepsilon_{\mathbf{k}_{F}}-\mu\right) \simeq-n_{c} / a \pi N$. Eqs. (167) yields

$$
N \mathscr{N}_{f}(0) Z_{f}^{-1}= \pm \frac{n_{c}}{a \pi} \frac{1}{\tilde{\omega}_{f}}
$$

where the upper (lower) sign applies to $\mathrm{Ce}$ and $\mathrm{Eu}(\mathrm{Yb})$ compounds in which $\tilde{\omega}_{f}>0$ $\left(\tilde{\omega}_{f}<0\right)$ and the Kondo resonance is above (below) the chemical potential.

Since the specific heat coefficient of the periodic Anderson model in the QP approximation is given by

$$
\gamma=N \frac{\pi^{2} k_{B}^{2}}{3} \sum_{\mathbf{k}} \delta\left(\mu-\Omega_{\mathbf{k}}^{ \pm}\right)=N \frac{\pi^{2} k_{B}^{2}}{3}\left[\mathscr{N}_{c}(0)+\mathscr{N}_{f}(0) Z_{f}^{-1}\right]
$$

we have the FL relationship

$$
\gamma=\frac{\pi^{2} k_{B}^{2}}{3} \frac{1}{T_{K}} \simeq \frac{\pi^{2} k_{B}^{2}}{3} \frac{n_{c}}{a \pi \tilde{\omega}_{f}}
$$


where we assumed that the enhanced $f$ contribution dominates. The Kondo temperature $T_{K} \simeq 1 / \gamma$ is thus related to the center of the Kondo resonance $\tilde{\omega}_{f}$. The numerical coefficient $a$ can be obtained from the NRG solution of the DMFT equations, which can provide the numerical values of $\gamma$ and $\tilde{\omega}_{f}$.

The width of the Kondo resonance $\tilde{\Delta}_{f}$. can be related to $\gamma$ by using Eqs. (170) and (171) which give at $\omega=0$,

$$
\frac{a N}{n_{c}}=\left|\frac{\tilde{\omega}_{f}}{\tilde{\Delta}_{f}}\right|+\left|\frac{\tilde{\Delta}_{f}}{\tilde{\omega}_{f}}\right| .
$$

Thus, $\left|\tilde{\omega}_{f} / \tilde{\Delta}_{f}\right|=\left(a N / 2 n_{c}\right) \pm \sqrt{\left(a N / 2 n_{c}\right)^{2}-1}=C$. The negative sign gives $\tilde{\Delta}_{f} \simeq$ $\left(N \pi k_{B}^{2}\right) /\left[3 \gamma\left(1+C^{2}\right)\right]$, such that for $N / n_{c} \gg 1$ and $C \simeq n_{c} / a N$ we have $\tilde{\Delta}_{f} \simeq N \pi k_{B}^{2} / 3 \gamma$.

To estimate the imaginary part of $\Sigma_{f}(\omega)$, which is also needed for thermal transport, we use the diagrammatic analysis. In infinite dimensions this gives the FL expression $[56,51]$

$$
-\operatorname{Im} \Sigma_{f}(\omega, T) \simeq \frac{\pi}{2}\left[\omega^{2}+\left(\pi k_{B} T\right)^{2}\right](N-1) \mathscr{N}_{f}^{3}(0) \Gamma_{f}^{2},
$$

where $\Gamma_{f}$ is the irreducible 4-point scattering vertex for electrons with different flavors. Eq. (174) is a straightforward generalization of the result given by the 2 nd order perturbation theory in which the bare interaction $U_{f f}$ is replaced by the $\Gamma$-vertex. In the limit of large correlations, when the charge fluctuations are suppressed, the Ward identity $[56,57]$ gives $Z_{f}^{-1}=(N-1) \mathscr{N}_{f}(0) \Gamma_{f}$, such that $\gamma=\left(\pi^{2} k_{B}^{2} / 3\right) N(N-1) \mathscr{N}_{f}^{2}(0) \Gamma_{f}$ and

$$
-(N-1) \mathscr{N}_{f}(0) \operatorname{Im}\left[\frac{\partial^{2} \Sigma_{f}(\omega)}{\partial \omega^{2}}\right]_{\omega=0^{+}} \simeq \frac{9 \gamma^{2}}{\pi^{3} k_{B}^{4} N^{2}} \simeq \frac{1}{\pi \tilde{\Delta}_{f}^{2}} .
$$

The transport relaxation time can now be obtained by substituting $\Sigma_{c}\left(\omega^{+}, T\right)$ in Eq. (157) which yields

$$
\tau(\omega, T) \simeq \frac{\left[\omega-\tilde{\varepsilon}_{f}(\omega, T)+\mu\right]^{2}+\left[\operatorname{Im} \Sigma_{f}\left(\omega^{+}, T\right)\right]^{2}}{V^{2}\left[\delta-\operatorname{Im} \Sigma_{f}\left(\omega^{+}, T\right)\right]} .
$$

The singularity at $T=0$ is avoided by taking the limit $\omega \rightarrow 0$ before $\delta \rightarrow 0$.

The transport coefficients of the periodic $S U(N)$ Anderson model in the FL regime are obtained by making the lowest order Sommerfeld expansion of Eq. (153), which gives

$$
L_{m n}=\left[\omega^{m+n-2} \Lambda(\omega, T)\right]_{\omega=0}+\frac{\pi^{2} k_{B}^{2} T^{2}}{6}\left\{\frac{\partial^{2}}{\partial \omega^{2}}\left[\omega^{m+n-2} \Lambda(\omega)\right]\right\}_{T, \omega=0}
$$

The correlation function $\Lambda(\omega)$ is obtained by substituting for $\mathscr{N}_{c}(\omega)$ and $\tau(\omega, T)$ the expressions derived above. Neglecting the energy dependence of $\mathscr{N}_{c}^{0}(\omega)$ for $\omega \simeq 0$ we obtain for the electrical resistance of $N$ parallel channels the result,

$$
\rho(T) \simeq \frac{\left(1+C^{2}\right)^{2}}{(N-1) N} \frac{3 \mathscr{V} a^{2} D^{2}}{5 \hbar e^{2} v_{k_{F}}^{2} n_{c}^{2}}\left(\frac{3 \gamma T}{\pi k_{B}}\right)^{2},
$$


where we approximated $N \mathscr{N}_{c}(0) \simeq n_{c} / \pi a D$ in Eq.(168) or (167) and used for Im $\Sigma_{f}(\omega, T)$ the expression Eq. (174). When $N / n_{c} \gg 1$ and $C \ll 1$, the FL law reads[51]

$$
\rho(T) \simeq A \frac{\gamma^{2} T^{2}}{(N-1) N n_{c}^{2}}\left(\frac{3}{\pi k_{B}}\right)^{2}=\frac{A \pi^{2}}{(N-1) N}\left(\frac{T}{T_{K}}\right)^{2},
$$

where $A=3 \mathscr{V} D^{2} /\left(5 \hbar e^{2} v_{k_{F}}^{2}\right)$.

The Seebeck coefficient is given by

$$
\frac{\alpha(T)}{T}=-\frac{\pi^{2}}{3} \frac{k_{B}^{2}}{|e|}\left\{\frac{1}{\mathscr{N}_{c}(\omega)} \frac{\partial \mathscr{N}_{c}(\omega)}{\partial \omega}+\frac{1}{\tau(\omega)} \frac{\partial \tau(\omega)}{\partial \omega}\right\}_{\omega, T=0}
$$

and we shall neglect the first term assuming $d\left[\ln \mathscr{N}_{c}(\omega)\right] / d \omega \ll d[\ln \tau(\omega)] d \omega$ for a slowly varying $\mathscr{N}_{c}^{0}(\omega)$. Equations (167), (171), (174), and (176) give $d[\ln \tau(\omega)] /\left.d \omega\right|_{\omega=0} \simeq \mp 2 a \pi N \mathscr{N}_{f}(0) / n_{c} Z_{f}$, such that the low-temperature Seebeck coefficient becomes $[60,61]$

$$
\alpha(T)= \pm \frac{2 a \pi}{|e|} \frac{\gamma T}{n_{c}}= \pm \frac{2 \pi^{2}}{3} \frac{k_{B}}{|e|} \frac{T}{T_{K}} .
$$

The upper (lower) sign applies to $\mathrm{Ce}$ and $\mathrm{Eu}(\mathrm{Yb})$ systems and $T_{K} / k_{B}=a \pi \tilde{\omega}_{f} / n_{c}$ defines the lattice Kondo scale.

The Sommerfeld expansion also gives $L_{22} / L_{11}=\pi^{2} k_{B}^{2} T^{2} / 3$ and $L_{12} / L_{11}=$ $\mp 2 a \pi \gamma T^{2} / n_{c}$, so that the thermal conductivity becomes

$$
\kappa(T)=\mathscr{L}_{0} T \sigma(T)\left[1-\frac{4 \pi^{2}}{3}\left(\frac{T}{T_{K}}\right)^{2}\right],
$$

The square bracket provides corrections to the WF law. In normal metals, $T_{K}$ should be replaced by $E_{F} / k_{B} \gg T$ and the WF law holds. In correlated systems, $T_{K}$ can be small and the deviations from the WF law can be large (although Eqn. (181) only holds for $\left.T<<T_{K}\right)$.

The renormalization of the average velocity in Eq.(178) offers a possible explanation of the sharp and asymmetric maximum of the $A$ coefficient which is observed in resistivity measurement of $\mathrm{Ce}$ intermetallics under pressure [62]. Consider, for example, the PAM with 3 doublets split by the CF. At low temperatures the scattering of c-electrons on two lowest doublet gives rise to the Kondo resonances which accommodate most of the f-charge, i.e., there are $n \simeq n_{c}+1 / 2$ electrons in each of the resonant channels. The 4 excited $f$ states give rise to simple scattering with constant phase shift. These nonresonant channels have $n \simeq n_{c}$ electrons; they contribute to the residual resistivity but not to the temperature dependent part of the resistivity.

The heavy mass is found in two resonant channels with $n=n_{c}+1 / 2$ electrons, provided $\mathbf{k}_{F}$ is close to the edge of the BZ. But this makes $v_{K_{F}}^{2}$ quite small and gives rise to the T-dependent resistivity, $\rho(T) \simeq \rho_{0}+\tilde{A} T^{2}$. In Ce compounds, heavy mass is seen in systems with large $\tilde{A}$. In these systems, pressure gives rise to a further increase of $\tilde{A}(P)$, which might be due to the reduction of $v_{k_{F}}^{2}$, because pressure increases hybridization and 
shifts the QP bands further apart. Since $\mathbf{k}_{F}$ is fixed by the Luttinger theorem, pressure reduces the dispersion and $v_{k_{F}}^{2}$. Of course, the reduction of $n_{f}$ enhances $T_{K}$ but for $\mu$ close to the band edge, the change in $n_{f}$ cannot be large and $T_{K}$ might not increase sufficiently to compensate the reduction of $v_{k_{F}}^{2}$. This might explain the initial increase of $\tilde{A}$ with pressure.

Above some critical pressure, the system becomes a valence fluctuator, i.e., the degeneracy of the f-state increases and the CF excitations vanish. All the f-channels are now equivalent and single f-electron is distributed over 6 channels. There are $n_{c}+1 / 6$ electrons in each channel and $\mathbf{k}_{F}$ is shifted away from the edge of the BZ. The FS average of $v_{K_{F}}^{2}$ and the characteristic energy are both enhanced, which reduces the coefficient $\tilde{A}$, as seen in Wilhelm's data [46]. A further increase of pressure increases the characteristic temperature, without changing $v_{K_{F}}^{2}$, i.e., above the critical pressure $A$ decreases. Thus, $\tilde{A}(P)$ exhibits an asymmetric maximum.

\section{The high-temperature 'poor man's' solution}

At elevated temperature, the FL laws breaks down and to obtain $\alpha(T)$ we need the full solution of the periodic Anderson model with the CF splittings and/or large degeneracy. Such a solution is not available at present and to estimate $\alpha(T)$ we use a 'poor man's' approach. We assume that the conduction electrons scatter incoherently on the $4 f$ ions and calculate the transport relaxation time in the T-matrix approximation. We write $\Sigma_{c}\left(k, \omega^{+}\right) \simeq n_{i} T_{k k}\left(\omega^{+}\right)$, where $T_{k k}\left(\omega^{+}\right)$is the single-ion scattering matrix on the real axis, and for the stoichiometric compounds set the concentration of $4 f$ ions to $n_{i}=1$. Since transport integrals are restricted to the Fermi window, we average $T_{k k}\left(\omega^{+}\right)$over the FS and calculate $\tau(\omega)$ using Eq. (157). However, for a complete description, the crystal field (CF) splitting has to be taken into account.

In the case of a single scattering channel (no CF splitting) the vertex corrections to the T-matrix vanish by symmetry and the conduction electron's self energy in Eq. (157) is given by $\Sigma_{c}\left(\omega^{+}\right)=V^{2} G_{f}\left(\omega^{+}\right)$, where $G_{f}\left(\omega^{+}\right)$is the retarded Green's function of the effective $N$-fold degenerate single impurity Anderson model. When the degeneracy is lifted by the CF splitting the vertex corrections do not vanish but we neglect them anyway and use

$$
\Sigma_{c}\left(\omega^{+}\right)=\sum_{\Gamma} V_{k_{F} \Gamma} G_{\Gamma}\left(\omega^{+}\right) V_{\Gamma k_{F}},
$$

where $V_{\Gamma k_{F}}=\langle\Gamma|V| \mathbf{k}\rangle$ is the FS average of the matrix element for the scattering between the $c$-electrons (in the $\mathbf{k}$-state) and the $f$-state (belonging to the irrep $\Gamma$ ) and $G_{\Gamma}\left(\omega^{+}\right)$ is the corresponding Green's function of the single impurity Anderson model with CF splitting.

As regards the validity of the 'poor man's' approach we point out that the DMFT+NRG solution[63] of the spin-1/2 Anderson lattice shows that the electrical resistance $\rho(T)$ increases rapidly and is very large at temperature $T_{K}$ where $\alpha(T)$ has a maximum. For $T \geq T_{K} / 2$, the thermopower of the lattice model[63] is very similar to the exact results[64] obtained for the spin-1/2 Anderson impurity. This indicates that the 'poor man's' approach can be used to describe the stoichiometric compounds at 
temperatures above $T_{K} / 2$, provided the single impurity scattering is solved by methods which can deal with large Coulomb correlation and the CF splitting.

The experimental results on the heavy Fermion and valence fluctuators provide additional support for the 'poor man's' approach. The data show that the residual resistance of ternary and quaternary compounds like $\mathrm{EuCu}_{2}\left(\mathrm{Ge}_{1-x} \mathrm{Si}_{x}\right)_{2}, \mathrm{CePt}_{1-x} \mathrm{Ni}_{x}$ and $\mathrm{YbIn}_{1-x} \mathrm{Ag}_{x} \mathrm{Cu}_{4}$ grows rapidly with $x$ and for $0.3 \leq x \leq 0.8$ the mean free path is reduced by disorder to about a single lattice spacing. In these random alloys the electron propagation is incoherent even at $T=0$ and the single impurity model should apply down to the lowest temperatures. At low doping and in stoichiometric compounds, the impurity description breaks down at low temperatures where $\rho_{0}$ is small. However, in these systems $\rho(T)$ and $\alpha(T)$ grow rapidly with increasing temperature (RT) and attain large maxima at $T_{K}^{\rho}$ and $T_{K}$, respectively. In the case of degenerate $f$-states or small CF splitting, the data show $[65,66,67] T_{K}^{\rho}<T_{K}<R T$ and for $T \geq T_{K}^{\rho} / 2$ there is not much difference between the stoichiometric compounds and doped systems, i.e., the impurity model applies. For large CF splitting, $\rho(T)$ has two maxima: a low-temperature one at $T_{K}^{\rho}$ and a high-temperature one at $T_{\rho}$. The thermopower also exhibits two maximal: a low-temperature one at $T_{K}>T_{K}^{\rho}$ and a high-temperature one at $T_{S}$. For $T \geq T_{K} / 2$, where the mean free path is short, the thermopower of periodic systems exhibits the same qualitative features as in random alloys, and can be explained in terms of impurity scattering. The experimental data show that the functional form of $\alpha(T)$ is strongly affected by pressure or chemical pressure. The fact that all the qualitative features of the pressureinduced variations of $\alpha(T)$ can be successfully explained by impurity scattering justifies, a posteriori, the 'poor man's' approach.

The thermoelectric properties of the single impurity Anderson model with the CF split $f$-states are obtained by the NCA, which is explained in detail in Refs. [68] and [69]. In the limit of large asymmetry and infinite $f-f$ correlation, the Kondo temperature $T_{K}$, obtained from the low-energy peak of the spectral function[70], agrees with the exact result[71], $T_{K}=3 \gamma / \pi k_{B} \cdot \gamma=\left(\pi^{2} k_{B}^{2} / 3\right) N \mathscr{N}_{f}(\mu) Z_{f}^{-1}$ is the impurity contribution to the specific heat coefficient, $\mathscr{N}_{f}(\mu)=(1 / \pi \Gamma) \sin ^{2}\left(\pi n_{f} / N\right)$ is the $f$-DOS at the Fermi level, and $Z_{f}$ is the renormalization factor. For a structureless $c$-DOS, the NCA shows that $T_{K}$ depends sensitively on the degeneracy of the $f$-state. A small $T_{K}$ is found for small $N$ and large $\Delta_{C F}$, while a large $T_{K}$ is found for large $N$ and small $\Delta_{C F}$. For a given $N$ and $\Delta_{C F}$, the Kondo scale is a monotonic function of $n_{f}$. It has a minimum at $n_{f}=1$ and increases rapidly as $n_{f}$ is reduced. In what follows, we discuss the behavior of $\alpha(T)$ assuming that pressure or doping increase the coupling constant $g$ and reduce $n_{f}$ but do not change $\Delta_{C F}$.

We consider first a $N$-fold degenerate $f$-state and show typical NCA results $[68,69,61]$ in Fig. 1, where thermopower is plotted as a function of temperature for several values of $E_{f}$. The calculations are performed for a half-filled, semielliptic $c$-band, no CF splitting $(N=8)$, and a constant hybridization $\Gamma$. A decrease of $E_{f}$ gives rise to an increase of $g$, which mimics the effect of pressure in Eu intermetallics. The thermopower is characterized by the Kondo maximum $\alpha_{S}$ at temperature $T_{S} \simeq T_{K}$. The high-temperature behavior depends strongly on the value of $n_{f}$. [Since $n_{f}$ can be temperature-dependent we characterize the system by $n_{f}\left(T_{K}\right)$ ]. For $n_{f} \simeq 1$, the thermopower has a large hightemperature slope, changes sign at $T_{0}>T_{K}$ and assumes large negative values above 
Thermopower S(T) reveals

characteristic features

$\mathrm{S}(\mathrm{T})$ is much larger than in normal metals.

$S(T)$ changes with pressure.

At each pressure $\mathrm{S}(\mathrm{T})$ assumes a typical shape.

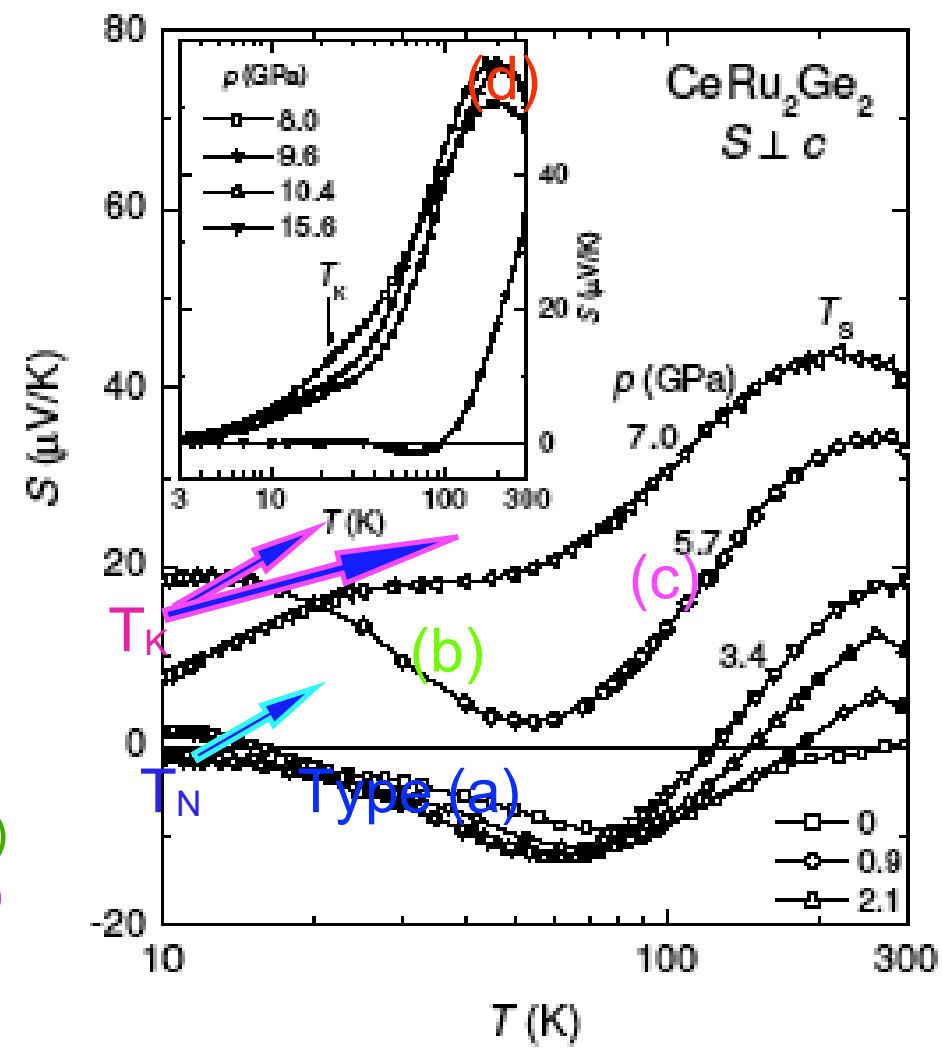

Figure 1: Temperature dependenes of the thermodectrie power $S(T)$ of

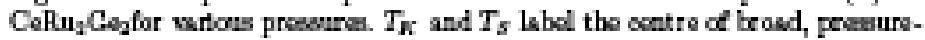
indwood maxima, rolated to the Koedo efoet and the crystalline elocitie fiald,

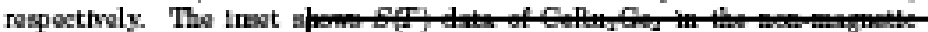
phase.

\section{CeRu Ge example}

FIGURE 1. Thermopower of the single impurity Anderson model of a 8-fold degenerate $f$-state calculated by the NCA for fixed hybridization $\Gamma=0.015 \mathrm{eV}$ is plotted as a function of temperature for several values of $E_{f}$, as indicated in the figure. The values of $n_{f}\left(T_{K}\right)$ are $0.76,0,81,0, .860 .91$, and 0.93 for $-E_{f}=0.12,0.15,0.18,0.22$, and 0.25 , respectively.

$T_{0}$. The $T_{S}$ and $T_{0}$ increase, and the high-temperature slope of $\alpha(T)$ decreases with decreasing $n_{f}$. For $n_{f}<0.7$, we still find a shallow maximum of $\alpha(T)$ below RT but the high-temperature slope is very small and the sign-change does not occur. A similar behavior is obtained if the coupling constant $g$ is reduced by increasing $\Gamma$. For smaller $N$, we find the same qualitative features but $\alpha_{S}$ and $T_{S}$ are reduced; for $N=2$ and $n_{f} \simeq 1$, the asymmetry of the model is much reduced and the Kondo maximum is almost completely suppressed. 
The CF splitting leads to additional features which we explain by the example of an $f$ ion with two CF states separated by $\Delta_{C F}$. The respective degeneracies of the ground and the excited state are $M$ and $M^{\prime}$, where $M+M^{\prime}=N$. The system now has two characteristic low-energy scales: the Kondo temperature $T_{K}$ and a larger scale $T_{K}^{N} \gg T_{K}$ which comes into play[72] when the excited CF states become significantly populated at temperature $T_{\Delta} \simeq \Delta / 2$. For $T \geq T_{\Delta}$, the thermopower can be approximated by the function $\alpha_{N}(T)$ which describes an effective $N$-fold degenerate $f$-state with Kondo temperature $T_{K}^{N}$ and exhibits all the features discussed in the previous paragraph. For $T<T_{\Delta}$, the excited CF states are unoccupied and the properties are determined by the lowest $\mathrm{CF}$ state which is $M$-fold degenerate (typically, $M \ll N$ ). Thus, the thermoelectric response of a CF split $f$-level is described at low temperatures by an effective $M$-fold degenerate Anderson model with Kondo scale $T_{K}$. All other parameters being the same, the main difference between this effective model and a simple $M$-fold degenerate model with Kondo scale $T_{K}^{M}=\lim _{\Delta \rightarrow \infty} T_{K}$ is that $T_{K} \gg T_{K}^{M}$. The enhancement of $T_{K}$ is due to the virtual transitions from the ground to the excited CF states. The function $\alpha_{M}(T)$ which approximates $\alpha(T)$ at low temperatures exhibits all the usual Kondo features. For $n_{f} \simeq 1$, it has a Kondo maximum at $T_{K}$ and changes sign at $T_{0}>T_{K}$; in the case of a doublet ground state the Kondo maximum is very small and $\alpha(T)$ is negative down to the lowest accessible temperatures. For $0.7 \leq n_{f} \leq 0.95$, the Kondo maximum is enhanced with respect to $n_{f} \simeq 1$, the high-temperature slope of $\alpha(T)$ is reduced, and the signchange shifted to $T_{0} \gg T_{K}$. For $n_{f} \leq 0.7$ the maximum of $\alpha(T)$ is further enhanced but the sign-change is absent. Of course, for $T \geq T_{\Delta}$ the excited CF states come into play and $\alpha_{M}(T)$ ceases to be physically relevant.

These effects are illustrated for a ground state doublet and an excited quartet in Fig. 2, where $\alpha(T)$ is plotted as a function of temperature for various values of $\Gamma(p)$. The $\mathrm{CF}$ spitting $\Delta_{C F}$ is the same for all the curves. An increase of pressure increases $\Gamma(p)$ and $g(p)$, and reduces $n_{f}$, which has a drastic effect on the functional form of $\alpha(T)$. We assume at ambient pressure $n_{f} \simeq 1$ and choose $\Gamma(0)$ and $g(0)$ such that $\alpha(T)<0$ at RT. The corresponding NCA spectral function has well defined CF excitations [68, 69] which show that the low-energy scales satisfy $T_{K} \ll T_{K}^{N} \ll T_{\Delta}$. >From the previous discussion we expect $\alpha(T)$ with a very weak Kondo maximum at $T_{K}$, a sign-change at $T_{0}>T_{K}$, and large negative values for $T \gg T_{0}$. Nothing particular happens at $T \simeq$ $T_{K}^{N}$, where the excited CF states are still unoccupied. At $T_{\Delta}$, the excited CF states become thermally populated and the functional form of $\alpha(T)$ changes from $\alpha_{M}(T)$ to $\alpha_{N}(T)$. Thus, a system with a low Kondo scale and large CF splitting has $\alpha(T)$ with a negative maximum around $T_{\Delta}$ and a deep negative minimum at low temperatures. The thermopower of that shape is illustrated by $\Gamma=0.08$ and $\Gamma=0.10$ curves in Fig. 2, classified[11, 69] as type (a).

If $\Gamma(p)$ and $g(p)$ increase, such that $0.8 \leq n_{f} \leq 0.95$, the NCA shows that the lowenergy CF excitations are still resolved and $T_{K}(p)<T_{K}^{N}(p)<T_{\Delta}$. In this parameter range the Kondo maximum of $\alpha(T)$ is enhanced and shifted to higher temperatures, such that $\alpha_{M}(p, T)>\alpha_{M}(T)$ for $T \geq T_{K}(p)$. The values of $T_{K}, T_{0}(p)$ and $T_{K}^{N}(p)$ are much closer to $T_{\Delta}$ than at ambient pressure, and $T_{0}^{N}(p)$ is now above $T_{\Delta}$. Thus, at the crossover we have $\alpha_{M}(p, T)<0$ and $\alpha_{N}(p, T)>0$. Since $\alpha_{N}\left(p, T_{\Delta}\right)$ is enhanced with respect to the $p=0$ values, pressure enhances the Kondo maximum and brings it closer to the CF 


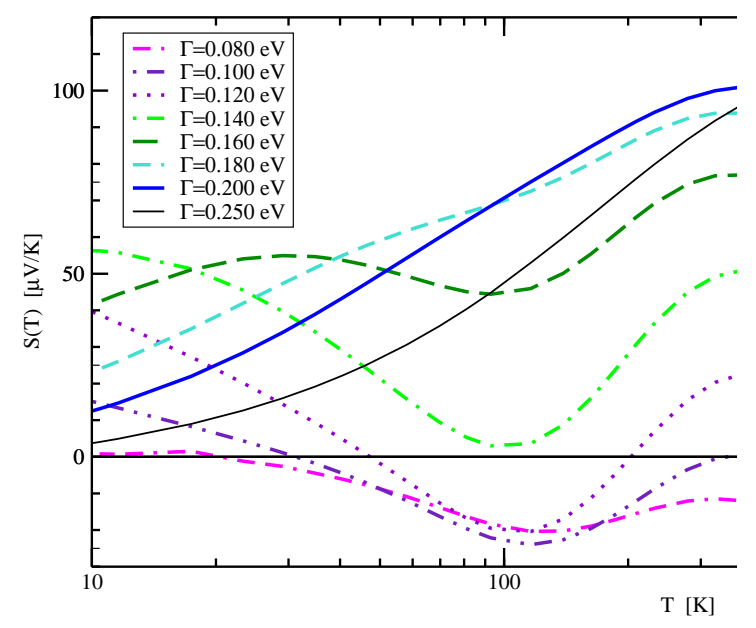

FIGURE 2. Thermopower of an $f$-ion with the ground state doublet and excited quartet calculated by the NCA for the CF splitting $\Delta=0.07 \mathrm{eV}$ is plotted as a function of temperature for several values of the hybridization strength $\Gamma$, as indicated in the figure. The two bottom curves describe the type (a) Kondo system, the third curve from the bottom is type (b), the two middle curves are type (c) and the third one from the top describes the type (d) Kondo systems. The two upper curves are type (e) and describes valence fluctuators.

maximum. The temperature interval in which $\alpha(T)<0$ shrinks with pressure, while $T_{S}$ does not change. These features are demonstrated by the $\Gamma=0.12$ curve in Fig. 2, which is classified as type (b).

In the pressure range such that $0.75 \leq n_{f} \leq 0.8$ the sign-change of $\alpha_{M}(T)$ is pushed to even higher temperatures and at large enough pressure $(\Gamma)$ we have eventually $T_{0}(p) \geq$ $T_{\Delta}$. The doublet-sextet crossover starts from $\alpha_{M}\left(T_{\Delta}\right) \geq 0$ and $\alpha(T)$ still exhibits two well resolved peaks but is always positive. These features are demonstrated by the $\Gamma=0.14$ and $\Gamma=0.16$ curves in Fig. 2, which are classified as type (c). A further increase of pressure gives $0.7 \leq n_{f} \leq 0.75$, which brings $T_{K}(p)$ so close to $T_{\Delta}$ that the Kondo and the CF peak cannot be resolved any more. The $\alpha(T)$ exhibits a single peak with a shoulder on the low-temperature side, as shown by the $\Gamma=0.18$ curve in Fig. 2 , which is classified as type (d). Note, as long as the low-energy CF excitations are well defined, the thermopower has a peak at temperature $T_{S} \simeq T_{\Delta}$ and the magnitude of this peak increases with pressure.

If $\Gamma(p)$ and $g(p)$ become very large and $n_{f}$ drops below 0.7 , the spectral function does 
not show the CF excitations any more. The thermopower acquires a single maximum at $T_{S}$ which is unrelated to $\Delta_{C F}$. The difference with respect to Kondo systems is that pressure shifts this thermopower peak to higher temperatures without changing its magnitude. Such behavior is typical of valence fluctuators and is demonstrated by the $\Gamma=0.20$ and $\Gamma=0.25$ curves in Fig. 2, which are classified as type (e). The curves in Fig. 1, which describe the system without the CF splitting such as Eu intermetallics, are also of this type. The $\mathrm{Yb}$ systems are characterized by an $f$-hole and the qualitative features of $\alpha(T)$ are obtained by reflecting ('mirror imaging') the curves in Figs. 1 or 2 on the temperature axis.

\section{Falicov-Kimball model}

The thermoelectric properties, the anomalous magnetic response of $f$-electrons and the metal-insulator transition of the conduction states that accompanies the valence-change transition in $\mathrm{YbInCu}_{4}$-like intermetallic compounds are very well described by the spindegenerate Falicov-Kimball model. The model considers a lattice of localized $f$-sites, which can be either occupied or empty, and conduction states which are delocalized via a nearest-neighbor hopping. The two types of electrons interact via a short-range Coulomb interaction and share a common chemical potential, which controls the total number of electrons $n=n_{c}+n_{f}$. The occupation of the $f$-states, which can be split into several CF levels, is restricted to $n_{f}<1$. For a given total number of electrons, thermal fluctuations can change the average $f$-occupation by transferring electrons or holes from the conduction band to the $f$-states and vice versa. The interaction has to be large enough to open a gap in the conduction band. We also assume that at low temperatures $\mu$ is within the lower (or upper) Hubbard band, so that the ground state is metallic. Since the model neglects quantum fluctuations, the ground state has no $f$-holes and the conduction electrons are essentially free.

The Hamiltonian of the spin-one-half Falicov-Kimball model can be written as[73]

$$
H=-\frac{t^{*}}{2 \sqrt{d}} \sum_{\langle i, j\rangle \sigma} c_{i \sigma}^{\dagger} c_{j \sigma}+E_{f} \sum_{i} w_{i}+U \sum_{i \sigma} w_{i} c_{i \sigma}^{\dagger} c_{i \sigma},
$$

where $c_{i \sigma}^{\dagger}\left(c_{i \sigma}\right)$ is the electron creation (annihilation) operator for an electron at site $i$ with spin $\sigma, E_{f}$ is the energy level of the localized electrons, $w_{i}$ is a variable that equals zero or one and corresponds to the localized electron number, and $U$ is the interaction strength. The hopping integral is scaled with the spatial dimension $d$ so as to have a finite result in the limit [74] $d \rightarrow \infty$; we measure all energies in units of $t^{*}=1$. We work on a hypercubic lattice where the noninteracting density of states is a Gaussian $\rho(\varepsilon)=\exp \left(-\varepsilon^{2}\right) / \sqrt{\pi}$.

The Falicov-Kimball model can be solved exactly by employing dynamical mean field theory $[75,76,77]$. Because the self energy $\Sigma(z)$ is local, the local Green's function satisfies

$$
G(z)=\int d \varepsilon \rho(\varepsilon) \frac{1}{z+\mu-\Sigma(z)-\varepsilon},
$$


with $z$ anywhere in the complex plane (we suppress the spin index here). The self energy, local Green's function, and effective medium $G_{0}$ are related by

$$
G_{0}^{-1}(z)-G^{-1}(z)=\Sigma(z)
$$

and the Green's function also satisfies

$$
G(z)=\left(1-w_{1}\right) G_{0}(z)+w_{1} \frac{1}{G_{0}^{-1}(z)-U} .
$$

Here $w_{1}$ is the average concentration of localized electrons,

$$
w_{1}=2 \exp \left[-\beta\left(E_{f}-\mu\right)\right] Z_{\uparrow}(\mu-U) Z_{\downarrow}(\mu-U) / Z,
$$

with $Z=Z_{\uparrow}(\mu) Z_{\downarrow}(\mu)+2 \exp \left[-\beta\left(E_{f}-\mu\right)\right] Z_{\uparrow}(\mu-U) Z_{\downarrow}(\mu-U)$ and

$$
Z_{\sigma}(\mu)=2 e^{\beta \mu / 2} \prod_{n} \frac{i \omega_{n}+\mu-\lambda_{\sigma}\left(i \omega_{n}\right)}{i \omega_{n}}
$$

The factor of two arises from the spin degeneracy of the f-electrons and the constraint that no more than one f-electron is allowed on any site. The symbol $\lambda_{\sigma}(z)$ is defined from the effective medium via $\lambda_{\sigma}\left(i \omega_{n}\right)=i \omega_{n}+\mu-G_{0 \sigma}^{-1}\left(i \omega_{n}\right), \omega_{n}=\pi T(2 n+1)$ is the fermionic Matsubara frequency, and $\beta=1 / T$. The algorithm for determining the Green's function begins with the self energy set equal to zero. Then Eq. (184) is used to find the local Green's function. The effective medium is found from Eq. (185) and the localized electron filling from Eq. (187). The new local Green's function is then found from Eq. (186) and the new self energy from Eq. (185). This algorithm is repeated until it converges. The transport coefficients are obtained in the limit of infinite dimensions by substituting the exact conduction electron Green's function into Eq. (154) and evaluating the integrals for transport coefficients numerically[78, 77].

The properties of the model exhibit all the features seen in the high-temperature phase of the intermetllic with the valence-change transition. The calculations show that the degenerate $f$-states become fractionally occupied at finite temperature and that the additional paramagnetic entropy of these excited states competes for the free energy with the excitation energy, the kinetic energy of the conduction electrons, and the interaction energy[77]. This gives rise to a valence transition at a temperature $T_{V}$, such that a substantial number of electrons (in $\mathrm{Eu}$ compounds) or holes (in $\mathrm{Yb}$ compounds) are transferred from the conduction band to the $4 f$ ions. The onset of the $4 f$ paramagnetism is accompanied by the reconstruction of the interacting density of conduction states and the shift of $\mu$ into the gap. Below $T_{V}$ the entropy of the system is given by the entropy of the conduction states. Above $T_{V}$ the entropy is dominated by the contribution of the localized, paramagnetic states. At intermediate temperatures the behavior can be quite complex[78, 77], because both the degeneracy of the $f$-states and the number of charge carriers change at $T_{V}$. The large reduction of the $\alpha / \mathscr{S}$ ratio at $T_{V}$ is an indication of the reduction of Fermi volume.

As regards the thermal transport, the calculations show [78, 77] that the electrical resistance of the paramagnetic phase is large and has a maximum at a temperature 
$T^{*} \gg T_{V}$, which is of the order of the gap, or the pseudo-gap in the density of states. The thermopower obtained by the DMFT is weakly temperature dependent and its sign depends on the band filling. The maximum of $\alpha(T)$ is also at $T^{*}$. The dc-conductivity, thermopower, and electronic contribution to the thermal conductivity, obtained by the DMFT solution for $U=2$ and five different $w_{1}$ values are shown in Fig. 3 (for details see [77]). For these parameters, there is always a region of exponentially small DOS near the chemical potential at low temperature [but in this region $\tau(\omega)$ decreases only as a power law]. As the localized electron concentration $w_{1}$ moves away from 0.5 , the high-temperature thermopower increases in magnitude due to the asymmetry in the DOS (it must vanish at 0.5 due to particle-hole symmetry) and the low-temperature thermopower shows a sharp peak for fillings close to half filling (the sign is hole-like, because the DOS from the lower Hubbard band dominates the transport coefficients at low temperature); the dc conductivity and thermal conductivity both vanish at low $T$ due to the "gap" as well. The thermoelectric figure-of-merit $Z T=T \sigma_{d c} S^{2} / \kappa_{e l}$ is plotted in Fig. 4-we find it is larger than one at high $T$ for $w_{1}<0.22$, and for fillings close to half filling, there is a low-temperature spike in $Z T$ that can become larger than one over a narrow temperature range. The spike at low $T$ is due to the large peak in $S$ and the small thermal conductivity; but the phonon contribution to the thermal conductivity can sharply reduce $Z T$ if the phonon thermal conductivity is much larger than the electronic thermal conductivity (this all electronic calculation provides only an upper bound to ZT). The Lorenz number is also plotted in Fig. 4. It gets huge at half filling, but becomes more metallic $\left(\approx \pi^{2} / 3\right)$ as the filling moves further away from half filling. It is not a constant even at low temperature because the system is not a Fermi liquid.

The above discussion shows that the DMFT calculations for a parameter set which yields the valence change transition at $T_{V}=50 \mathrm{~K}$ and opens a pseudo-gap of the order of $T^{*} \simeq 500 \mathrm{~K}$, explains the main features of the magnetic susceptibility, the XPS data, and the optical conductivity of the stoichiometric compound $\mathrm{YbInCu}_{4}$ at temperatures above $T_{V}$. The calculated thermopower[78] is of the order of a few $\mu V / K$, and its sign is either positive or negative, depending on the band filling and the shape of the conduction band. The proximity of $\mu$ to the pseudo-gap would lead (in a non-interacting system) to a shallow minimum of $\alpha(T)$ at a temperature of the order of $T^{*}$, but in an interacting system, the valence-change transition destabilizes the semiconducting phase, and gives rise to a discontinuity of $\alpha(T)$ at $T_{V}$. The low-temperature FL state has a large characteristic temperature and $|\alpha(T)|$ is a linearly increasing function of temperature. Thus, a cusp or even a discontinuity appears in $\alpha(T)$ at $T_{V}$, in good agreement with the experimental data.

FIGURE 3. (a) DC conductivity, (b) thermopower, and (c) electronic contribution to the thermal conductivity, for the spinless FK model with $\rho_{e}=1-w_{1}$ and $U=2$. Five fillings are shown: (i) $w_{1}=0.5$ (solid line); (ii) $w_{1}=0.4$ (dashed line); (iii) $w_{1}=0.3$ (chain-dotted); (iv) $w_{1}=0.2$ (dotted); and (v) $w_{1}=0.1$ (chain-triple-dotted). From Freericks and Zlatic, 2003 [77] 
FIGURE 4. (a) Lorenz number $\mathscr{L} k_{B}^{2} / e^{2}=\kappa_{e l} / \sigma_{d c} T$ and (b) electronic thermoelectric figure of merit $Z T=T \sigma_{d c} S^{2} / \kappa_{e l}$ for the spinless FK model with $\rho_{e}=1-w_{1}$ and $U=2$. Five fillings are shown: (i) $w_{1}=0.5$ (solid line); (ii) $w_{1}=0.4$ (dashed line); (iii) $w_{1}=0.3$ (chain-dotted); (iv) $w_{1}=0.2$ (dotted); and (v) $w_{1}=0.1$ (chain-triple-dotted). From Freericks and Zlatic, 2003 [77]

\section{ACKNOWLEDGMENTS}

I am pleased to acknowledge many useful discussions with R. Monnier and J.K. Freericks. They have contributed substantially to the research activity I have presented in these lecture notes.

\section{REFERENCES}

1. H. B. Callen, Phys. Rev. 73, 1349 (1949).

2. J. M. Luttinger, Phys. Rev. 135, A1505 (1964).

3. C. A. Domenicali, Rev.Mod.Phys. 26, 237 (1954).

4. G. D. Mahan, Many-Particle Physics, Plenum, New York, 1981.

5. L. D. L. E. M. Lifshitz, and L. P. Pitaevskii, Electrodynamics of Continuous Media, Elsevier Butterworth-Heinemann, Oxford, 1984.

6. A. F. Ioffe, Semiconductor Thermoelements and Thermoelectric Cooling, Infosearch, London, 1954.

7. M. Jonson, and G. D. Mahan, Phys. Rev. B 21, 4223 (1980).

8. M. Jonson, and G. D. Mahan, Phys. Rev. B 42, 9350 (1990).

9. G. D. Mahan, Good Thermoelectrics, Academic Press, San Diego, 1997, vol. 51 of Solid State Physics, p. 81.

10. J. Sakurai, H. Takagi, S. Taniguchi, T. Kuwal, Y. Isikawa, and J.-L. Tholance, J. Phys. Soc. Japan Suppl. B 65, 49 (1996).

11. P. Link, D. Jaccard, and P. Lejay, Physica B 225, 207 (1996).

12. V. Zlatić, I. Milat, B. Coqblin, G. Czycholl, and C. Grenzbach, Phys. Rev. 68, 104432 (2003).

13. G. Sparn, W. Lieka, U. Gottwick, F. Steglich, and N. Grewe, J. Magn. Magn. Mater. 47 \& 48, 521 (1985).

14. D. Jaccard, J. M. Mignot, B. Bellarbi, A. Benoit, H. F. Braun, and J. Sierro, J. Magn. Magn. Mater. $47 \& 48,23$ (1985).

15. D. Huo, T. Kuwai, T. Mizushima, Y. Isikawa, and J. Sakurai, Physica B 312 \& 313, 232 (2002).

16. P. B. van Aken, H. J. van Daal, and K. H. J. Buschow, Phys. Lett. A 49, 201 (1974).

17. R. Cibin, D. Jaccard, and J. Sierro, J. Magn. Magn. Mater. 108, 107 (1992).

18. D. Huo, K. Mori, T. Kuwai, S. Fukuda, Y. Isikawa, and J. Sakurai, Physica B 281 \& 282, 101 (2000).

19. J. Sakurai, D. Huo, D. Kato, T. Kuwai, Y. Isikawa, and K. Mori, Physica B 281 \& 282, 98 (2000).

20. H. Wilhelm, and D. Jaccard, Phys. Rev. 69, 214408 (2004).

21. K. Behnia, D. Jaccard, and J. Flouquet, J. Phys.: Condens. Matter 16, 5187 (2004).

22. F. Steglich, Festkoeorperprobleme 17, 319 (1977).

23. Y. Onuki, and T. Komatsubara, J. Magn. Magn. Mater. 63 \& 64, 281 (1987).

24. J. Sakurai, J. C. G. Sal, and J. R. Fernandez, J. Magn. Magn. Mater 140 - 144, 1223 (1995).

25. A. Amato, D. Jaccard, J. Sierro, P.Haen, P. Lejay, and J. Flouquet, J. Low. temp. Phys. 77, 195 (1989).

26. J. Sakurai, T. Ohyama, and Y. Komura, J. Magn. Magn. Mater. 47 \& 48, 320 (1985).

27. E. Bauer, Adv. Phys 40, 417 (1991).

28. D. Jaccard, J. M. Mignot, A. B. B. Bellarbi, H. F. Braun, and J. Sierro, J. Magn. Magn. Mater. 47 \& 48, 23 (1985).

29. C. Fierz, D. Jaccard, and J. Sierro, J. Appl. Phys. 63, 3899 (1988).

30. D. Jaccard, K. Behnia, and J. Sierro, Phys. Lett. A 163, 475 (1992).

31. Y. Bando, J. Sakurai, and E. V. Sampathkumaran, Physica B 186 - 188, 525 (1993). 
32. E. Gratz, E. Bauer, R. Hauser, N. Pillmayr, G. Hilscher, H. Mueller, and B. Barbara, J. Magn. Magn. Mater. 76 \& 77, 275 (1988).

33. J. Sakurai, H. Kamimura, and Y. Komura, J. Magn. Magn. Mater. 76 \& 77, 287 (1988).

34. D. Huo, K. Mori, T. Kuwai, H. Kondo, Y. Isikawa, and J. Sakurai, J. Phys. Soc. Japan 68, 3377 (1999).

35. M. Očko, C. Geibel, and F. Steglich, Phys. Rev. 64, 195107 (2001).

36. P. Link, D. Jaccard, and P. Lejay, Physica B 225, 207 (1996).

37. P. Link, D. Jaccard, , and P. Lejay, Physica B 223 \& 224, 303 (1996).

38. K. Alami-Yadri, D. Jaccard, and D. Andreica, J. Low Temp. Phys. 114, 135 (1999).

39. G. Nakamoto, T. Nobata, S. Ueda, Y. Nakajima, T. Fujioka, and M. Kurisu, Physica B 259 - 261, 154 (1999).

40. R. Casanova, D. Jaccard, C. Marcenat, N. Hamdaoui, and M. J. Besnus, J. Magn. Magn. Mater. 90 \& 91, 587 (1990).

41. O. Trovarelli, C. Geibel, B. Buschinger, R. Borth, S. Mederle, M. Grosche, G. Sparn, and F. Steglich, Phys. Rev. 60, 1136 (1999).

42. D. Andreica, K. Alami-Yadri, D. Jaccard, A. Amato, and A. Schenk, Physica B 259- 261, 144 (1999).

43. H. J. van Daal, P. B. van Aken, and K. H. J. Buschow, Phys. Lett. A 49, 246 (1974).

44. I. Aviani, M. Miljak, V. Zlatic, K.-D. Schotte, C. Geibel, and F. Steglich, Phys. Rev. 64, 184438 (2001).

45. H. Wilhelm, and D. Jaccard, Phys. Rev. 66, 064428 (2002).

46. H. Wilhelm, K. Alami-Yadri, B. Revaz, and D. Jaccard, Phys. Rev. B 69, 3651 (1999).

47. A. Demuer, A. T. Holmes, and D. Jaccard, J. Phys.: Condens. Matter 14, L529 (2002).

48. H. Wilhelm, D. Jaccard, V. Zlatić, R. Monnier, B. Delley, and B. Coqblin, J. Phys.: Condens. Matter 17, 823 (2005)

49. J. Sakurai, and Y. Isikawa, J. Phys. Soc. Japan 74, 1926 (2005).

50. K. Kadowaki, and S. B. Woods, Solid State Commun. 71, 1149 (1987).

51. H. Kontani, J. Phys. Soc. Japan 73, 515 (2004).

52. N. Tsujii, H. Kontani, and K. Yoshimura, Phys. Rev. Lett. 94, 057201 (2005).

53. A. L. Cornelius, J. M. Lawrence, , J. Sarrao, Z. Fisk, M. F. Hundley, G. H. Kwei, J. D. Thompson, C. H. Booth, and F. Bridges, Phys. Rev. B 56, 7993 (1997).

54. M. Očko, J. L. Sarrao, I. Aviani, D. Drobac, I. Ž ivković, and M. Prester, Phys. Rev. B 51, 975102 (2003).

55. J. L. Sarrao, Physica B 259 \& 261, 129 (1999).

56. K. Yamada, and K. Yosida, Prog. Theor. Phys. 76, 681 (1986).

57. K. Yamada, Electron Correlation in Metals, Cambridge University Press, Cambridge, 2004.

58. N. Vidhyadhiraja, and D. Logan, European Phys. Jour. B 39, 313 (2004).

59. D. E. Logan, and N. S. Vidhyadhiraja, J. Phys: Cond. Matter 17, 2935 (2005).

60. K. Miyake, and H. Kohno, J. Phys. Soc. Japan 74, 254 (2005).

61. V. Zlatić, R. Monnier, J. Freericks, and K. W. Becker, Phys. Rev. B 76, 085122 (2007).

62. H. Wilhelm, D.Jaccard, V. Zlatic, R. Monnier, B. Delley, and B. Coqblin, J. Phys: Cond. Matter 17, S830 (2005).

63. C. Grenzebach, F. B. Anders, G. Czycholl, and T. Pruschke, Phys. Rev. B 74, 195119 (2006).

64. T. Costi, A. Hewson, and V. Zlatić, J. Phys: Cond. Matter 6, 2519 (1994).

65. M. Kim, H. T. Y. Nakai, M. Sera, T. T. F. Iga, and S. Kunii, J. Phys. Soc. Japan 75, 064704 (2006).

66. S. Kobayashi, Y. Yoshino, S. Tsuji, M. Sera, and F. Iga, J. Phys. Soc. Japan 72, 25 (2002).

67. Z. Hossain, C. Geibel, N. Senthilkumaran, M. Deppe, M. Baenitz, F. Schiller, and S. L. Molodtsov, Phys. Rev. B 69, 014422 (2004).

68. N. E. Bickers, D. L. Cox, and J. Wilkins, Phys. Rev. B 36, 2036 (1987).

69. V. Zlatić, and R. Monnier, Phys. Rev. B 71, 165109 (2005).

70. B. Horvatić, and V. Zlatić, Phys. Rev. B 30, 6717-6731 (1984).

71. A. Hewson, The Kondo Problem to Heavy Fermions, Cambridge University Press, Cambridge, 1993.

72. K. Hanzawa, K. Yamada, , and K. Yosida, J. Magn. Magn. Mater. 47 \& 48, 357 (1985).

73. L. M. Falicov, and J. C. Kimball, Phys. Rev. Lett. 22, 997 (1969).

74. W. Metzner, and D. Vollhardt, Phys. Rev. Lett. 62, 324 (1989).

75. U. Brandt, and C. Mielsch, Z. Phys. B 75, 365 (1989).

76. U. Brandt, and C. Mielsch, Z. Phys. B 79, 295 (1990). 
77. J. K. Freericks, and V. Zlatić, Rev. Mod. Phys. 75, 1333 (2003).

78. J. K. Freericks, and V. Zlatić, Phys. Rev. B 64, 245118 (2001). 